\title{
Finding A Small Vertex Cover in Massive Sparse Graphs: Construct, Local Search, and Preprocess
}

\author{
Shaowei Cai \\ State Key Laboratory of Computer Science, \\ Institute of Software, Chinese Academy of Sciences, Beijing, China
}

Jinkun Lin

School of Electronics Engineering and Computer Science, Peking University, Beijing, China

\author{
Chuan Luo \\ Institute of Computing Technology, \\ Chinese Academy of Sciences, Beijing, China
}

SHAOWEICAI.CS@GMAIL.COM

JKUNLIN@GMAIL.COM

CHUANLUOSABER@GMAIL.COM

\begin{abstract}
The problem of finding a minimum vertex cover (MinVC) in a graph is a well known NPhard combinatorial optimization problem of great importance in theory and practice. Due to its NP-hardness, there has been much interest in developing heuristic algorithms for finding a small vertex cover in reasonable time. Previously, heuristic algorithms for MinVC have focused on solving graphs of relatively small size, and they are not suitable for solving massive graphs as they usually have high-complexity heuristics. This paper explores techniques for solving MinVC in very large scale real-world graphs, including a construction algorithm, a local search algorithm and a preprocessing algorithm. Both the construction and search algorithms are based on low-complexity heuristics, and we combine them to develop a heuristic algorithm for MinVC called FastVC. Experimental results on a broad range of real-world massive graphs show that, our algorithms are very fast and have better performance than previous heuristic algorithms for MinVC. We also develop a preprocessing algorithm to simplify graphs for MinVC algorithms. By applying the preprocessing algorithm to local search algorithms, we obtain two efficient MinVC solvers called NuMVC2+p and FastVC2+p, which show further improvement on the massive graphs.
\end{abstract}

\section{Introduction}

The proliferation of massive data sets has brought a series of computational challenges, as existing algorithms usually become ineffective on massive data sets, and for most problems we need to develop new algorithms. Many data sets can be modeled as graphs, and the study of massive realworld graphs, also called complex networks, grew enormously in the last decade. In this work, we consider the Minimum Vertex Cover (MinVC) problem and propose effective techniques for addressing this problem on massive graphs.

Given an undirected graph $G=(V, E)$, a vertex cover is a subset $S \subseteq V$, such that each edge in $G$ has at least one endpoint in $S$. Alternatively, a vertex cover is a set of vertices whose removal completely disconnects a graph. The MinVC problem requires us to find the minimum sized vertex cover in a graph. MinVC is a prominent combinatorial optimization problem with 
important applications, including network security, industrial machine assignment and applications in sensor networks such as monitoring link failures, facility location and data aggregation (Kavalci, Ural, \& Dagdeviren, 2014). It is also closely related to the Maximum Independent Set (MaxIS) problem, which has applications in social networks, pattern recognition, molecular biology and economics (Jin \& Hao, 2015).

There are important real-world tasks that call for solving MinVC on massive graphs, which mainly come from social networks and molecular biology. For example, consider the case where one has to select the minimum set of influential nodes in a social network such that some critical information is propagated to all nodes in the network in a single hop (or few hops). One solution of this problem is to determine an approximate MinVC of the network and use the nodes in MinVC for propagating information (Yadav, Sadhukhan, \& Rao, 2016). In the genetic analysis of gene transcription, the concept of vertex cover is used to determine the identity, proportion and number of transcripts connected to individual phenotypes and quantitative trait loci (QTL) regulatory models (Chesler \& Langston, 2005).

MinVC is a classical NP-hard problem and remains intractable even for cubic graphs and planar graphs with a maximum degree at most three (Garey \& Johnson, 1979). Furthermore, it is NP-hard to approximate MinVC within any factor smaller than 1.3606 (Dinur \& Safra, 2005), although one can achieve an approximation ratio of $2-o(1)$ (Karakostas, 2005).

\subsection{Previous Heuristics and Motivations}

Due to its NP-hardness, research into MinVC solving has been concentrated on heuristic algorithms for finding a "good" vertex cover in reasonable time. Heuristic algorithms for NPhard computational problems can be mainly divided into heuristic construction algorithms and local search algorithms (Hoos \& Stützle, 2004).

In the context of MinVC, construction algorithms generate a vertex cover by extending a partial solution, i.e., a vertex set. A construction algorithm for MinVC starts from an empty vertex set, and then iteratively adds vertices into the set, until it becomes a vertex cover. Two typical construction algorithms for MinVC include the maximal matching based algorithm, and a greedy construction algorithm which at each iteration adds the vertex that covers the most uncovered edges. These two algorithms are so classical that they are included in a well-known textbook of combinatorial optimization (Papadimitrious \& Steiglitz, 1982). However, construction algorithms alone do not provide good-quality solutions in practice, although they are of interest from a theoretical viewpoint. Due to this reason, practical work on construction algorithms for MinVC is rare. In practice, construction algorithms for MinVC are usually used to generate an initial solution for local search algorithms.

Local search is perhaps the most popular practical heuristic approach to NP-hard combinatorial optimization problems. Seen from the literature, a general scheme for local search algorithms for MinVC is as follows. It first uses a construction algorithm to obtain a vertex cover. Whenever it finds a vertex cover, it removes a vertex from the solution, and then iteratively performs small modifications to the candidate vertex set, such as removing a vertex, adding a vertex, or swapping a vertex pair, until the vertex set becomes a vertex cover. This process is repeated until a satisfactory solution is returned or a preset time limit is reached. There has been considerable interest in local search algorithms for MinVC in the last decade, e.g., (Richter, Helmert, \& Gretton, 2007; Andrade, Resende, \& Werneck, 2008; Pullan, 2009; Cai, Su, \& Sattar, 2011; Cai, Su, Luo, \& Sattar, 2013). In 
particular, a recent algorithm called NuMVC (Cai et al., 2013), which outperforms other heuristic algorithms on a broad range of benchmarks, makes a significant improvement in MinVC solving.

Previous local search algorithms for MinVC are mainly evaluated on randomly generated benchmarks and two benchmarks namely the DIMACS and BHOSLIB benchmark sets (Richter et al., 2007; Andrade et al., 2008; Pullan, 2009; Cai et al., 2011, 2013). The DIMACS and BHOSLIB are the two most popular benchmark sets for testing MinVC (also MaxIS and Maximum Clique) algorithms, as they are generally difficult to solve, and some DIMACS graphs arise from real-world applications. To improve the performance on these benchmarks, many sophisticated heuristics have been proposed and tested. Recent heuristics include max-gain vertex pair selection (Richter et al., 2007), edge weighting (Richter et al., 2007; Cai et al., 2011), $k$-improvement (also called $(k-1, k)$ swap) (Andrade et al., 2008), configuration checking (Cai et al., 2011), minimum loss removing and two-stage exchange (Cai et al., 2013). Most of the previous heuristics do not have sufficiently low complexity. Because the benchmark graphs used for testing previous algorithms are not large (usually with less than five thousand vertices), the complexity of heuristics did not show an obvious impact on the performance. However, for massive graphs where the size is much larger (e.g., with millions of vertices), the high complexity severely limits the ability of algorithms to handle these data sets.

Massive graphs call for new heuristics and algorithms. However, there is little work being done on heuristic algorithms for massive graphs. In particular, our work was the first research on local search for MinVC on massive graphs, when it was first presented in the IJCAI 2015 conference (Cai, 2015). We also study construction and preprocessing algorithms for MinVC. This work also shows that, when designing algorithms for solving problems on massive graphs, a key issue is on making a good balance between the time complexity and the effectiveness of heuristics.

\subsection{Main Contributions}

This paper focuses on solving massive sparse instances of the MinVC problem in practice. The main technical contributions of this paper are as follows.

1. We propose a new construction algorithm for MinVC called EdgeGreedyVC. We show theoretically that EdgeGreedyVC always returns a minimal vertex cover with a linear complexity. Also, experimental results on real-world massive graphs demonstrate that it achieves a good balance between solution quality and run time when we compare it with previous construction algorithms.

2. We propose a new local search algorithm for MinVC called FastVC. A novel technique in FastVC is a probabilistic heuristic named Best from Multiple Selections (BMS), which returns a good-quality vertex from a large set of candidate vertices with a very high probability. The BMS heuristic approximates the minimum loss removing heuristic (Cai et al., 2013) very well and lowers the complexity from $O(|V|)$ to $O(1)$. We carry out experiments to evaluate FastVC on massive real-world graphs, compared with a representative of the state of the art algorithm named NuMVC as well as its variant $\mathrm{NuMVC}_{e}$ which uses the same construction heuristic as FastVC. Experimental results show that FastVC finds significantly better quality vertex covers than NuMVC and $\mathrm{NuMVC}_{e}$ on most instances.

3. We improve two previous construction algorithms by adding a shrinking phase and by using an efficient data structure. Then we integrate all three construction algorithms into both 
NuMVC and FastVC, leading to two improved local search algorithms for MinVC named NuMVC2 and FastVC2.

4. We develop a two-phase preprocessing algorithm to simplify graphs for MinVC algorithms. Experimental results show that the preprocessing algorithm is effective and efficient. By applying the preprocessing algorithm, we further improve NuMVC2 and FastVC2 and develop two more efficient MinVC solvers called NuMVC2+p and FastVC2+p.

This paper is an extended and improved version of a conference paper (Cai, 2015). New contributions in this paper include parts of the first and second contributions (the study and comparison of construction algorithms, the experiments with $\mathrm{NuMVC}_{e}$ ), as well as the whole third and fourth contributions. Also, while experiments are only performed on some typical instances in the conference paper, experiments in this paper are performed on the complete set of benchmark instances.

\subsection{Structure of the Paper}

In the next section, we introduce some preliminary knowledge, including definitions and notation, preliminaries of local search for MinVC, as well as the benchmarks and experiment methodology in this work. In Section 3, we investigate previous construction algorithms for MinVC and propose a new construction algorithm called EdgeGreedyVC, and compare EdgeGreedyVC with previous construction algorithms. In Section 4, we describe the local search algorithm FastVC and present the key function based on the BMS heuristic, and carry out experiments to evaluate FastVC. In Section 5, we improve two previous construction algorithms and integrate all the three construction algorithms into both NuMVC and FastVC, leading to two improved local search algorithms named NuMVC2 and FastVC2. In section 6, we develop a preprocessing algorithm for MinVC and apply it to further improve NuMVC2 and FastVC2, leading to NuMVC2+p and FastVC2+p. Finally, we give some concluding remarks.

\section{Preliminaries}

In this section, we first introduce the basic definitions and natation that will be used in this paper, and then we give some preliminaries about local search for MinVC. Finally, we introduce the benchmarks and the experimental methodology that we use in our experiments.

\subsection{Basic Definitions and Notation}

An undirected graph $G=(V, E)$ consists of a vertex set $V$ and an edge set $E$ where each edge is a 2-element subset of $V$. For an edge $e=\{u, v\}$, we say that vertices $u$ and $v$ are the endpoints of edge $e$. For convenience of discussions on complexity, we define $n=|V|$ and $m=|E|$. Two vertices are neighbors if and only if they both belong to some edge. The neighborhood of a vertex $v$ is denoted as $N(v)=\{u \in V \mid\{u, v\} \in E\}$, and the closed neighborhood as $N[v]=\{v\} \cup N(v)$. The degree of a vertex $v$ is defined as $\operatorname{deg}(v)=|N(v)|$.

For an undirected graph $G=(V, E)$, a vertex cover of a graph is a subset of $V$ that contains at least one of the two endpoints of each edge. A vertex cover is minimal if taking any vertex out of it would make it not a vertex cover. An independent set is a subset of $V$ where no two vertices are neighbors. A vertex set $S$ is a vertex cover of $G$ if and only if $V \backslash S$ is an independent set of $G$. We 
are concerned in this paper with the problem of finding a vertex cover as small as possible (MinVC). Equivalently, this problem can be viewed as seeking as large an independent set as possible, which also has important applications.

Given an undirected graph $G=(V, E)$, a candidate solution for MinVC is a subset of vertices $X \subset V$. An edge $e \in E$ is covered by a candidate solution $X$ if at least one endpoint of $e$ belongs to $X$, and otherwise we say it is uncovered by $X$. For convenience, in the rest of this paper, we use $C$ to denote the current candidate solution. A vertex has two states: selected for covering (i.e., $v \in C$ ), or not selected (i.e., $v \notin C$ ). The age of a vertex is the number of steps since its state was last changed.

Given an undirected graph $G$ and a candidate solution $X$ for MinVC, for a vertex $v \in X$, the loss of $v$, denoted as $\operatorname{loss}(v, X)$, is defined as the number of covered edges that would become uncovered by removing $v$ from $X$; for a vertex $v \notin X$, the gain of $v$, denoted as $\operatorname{gain}(v, X)$, is defined as the number of uncovered edges that would become covered by adding $v$ into $X$. In this work, when talking about loss and gain of vertices, the candidate solution always refers to the current candidate solution $C$ and thus it is omitted. We write $\operatorname{loss}(v)$ and $\operatorname{gain}(v)$ for $\operatorname{loss}(v, C)$ and $\operatorname{gain}(v, C)$, for the sake of convenience. Both loss and gain are scoring properties of vertices.

\subsection{Preliminaries of Local Search for MinVC}

One popular way to solve the MinVC problem is based on iteratively solving its decision version - given a positive integer number $k$, searching for a $k$-sized vertex cover. The general scheme is as follows: At the beginning, a vertex cover is constructed; whenever the algorithm finds a vertex cover of $k$ vertices, one vertex is removed from the vertex cover ${ }^{1}$, and the algorithm starts from the resulting vertex set to search for a vertex set of $k-1$ vertices that covers all edges (i.e., a vertex cover of $k-1$ vertices) by performing local search. When the algorithm terminates, it outputs the smallest vertex cover it has found.

For local search MinVC algorithms that are based on iteratively solving the decision problem, each search step consists of exchanging a pair of vertices: a vertex $u \in C$ is removed from $C$, and a vertex $v \notin C$ is put into $C$. Such a step is called an exchange step. In the literature, there are two ways to perform an exchange step. The first one is adopted by algorithms before NuMVC, which chooses a vertex pair from candidate vertex pairs, and then exchanges them and updates scoring properties accordingly. The second method, proposed in NuMVC and named two-stage exchange, works in a "separate" fashion: it first chooses a vertex $u \in C$ and removes it, and updates scoring properties accordingly, and then chooses a vertex $v \notin C$ and adds it, and updates scoring properties accordingly.

\subsection{Benchmarks and Experiment Methodology}

In this work, in order to study the algorithms, we carry out extensive experiments and report the results in tables. In this subsection, we introduce the benchmarks, the experiment setup and reporting methodology, so that the readers can understand the experiment parts more easily.

For our experiments, we collected all undirected simple graphs (not including DIMACS and BHOSLIB graphs) we could find from the Network Data Repository online (Rossi \& Ahmed,

1. If after removing one vertex, the vertex set remains a vertex cover, then more vertices are removed until it is not a vertex cover. 
2015). ${ }^{2}$ All these graphs are generated from real-world applications. Many of these real-world graphs have millions of vertices and dozens of millions of edges, while at the same time being quite sparse. We calculate the density of each graph, i.e., $m /\left(\begin{array}{l}n \\ 2\end{array}\right)$, and the averaged density of these graphs is 0.00859 , while the maximum one is 0.347 . We also calculate the averaged degree $2 \mathrm{~m} / \mathrm{n}$ for each graph, and the averaged value of these figures is 26.15 , while the maximum one is 181.19. Some of these benchmarks have recently been used in testing algorithms for Maximum Clique and Coloring problems (Rossi \& Ahmed, 2014; Rossi, Gleich, Gebremedhin, \& Patwary, 2014; Wang, Cai, \& Yin, 2016; Cai \& Lin, 2016). There are 102 graphs in total in this suite of benchmarks. The graphs can be grouped into 11 classes, including biological networks, collaboration networks, interaction networks, infrastructure networks, Amazon recommendation networks, Tweeter networks, Facebook networks, scientific computation networks, social networks, technological networks, and web linkage networks, in the order of their appearance in the tables. There is also a group of temporal reachability networks, where the graphs are small (usually with several hundreds of vertices) and the algorithms find the same quality solution on all the graphs, and thus are not included in our experiments.

All the algorithms in our experiments, either developed in this work or not, are implemented in the $\mathrm{C}++$ programming language by their authors, and have been complied by g++ (version 4.4.5) with the '-O3' option for our experiments. All experiments are carried out on a workstation under Ubuntu Linux (version 14.04), using 2 cores of an Intel i7-4800MQ $2.5 \mathrm{GHz}$ CPU and 32 GByte RAM.

Most experiments in this work involve comparing the solution quality and run time of different MinVC algorithms. In particular, all local search algorithms (sometimes combined with a preprocessing algorithm) are executed 10 times with the same random seeds $(\{1,2, \ldots, 10\})$ on each instance with a time limit of 1000 seconds for each execution. For each algorithm on each instance, we report three metrics:

- The minimum size of vertex cover found by the algorithm among the 10 executions, denoted by 'min' in the tables.

- The averaged size of vertex covers found by the algorithm over the 10 executions, denoted by 'avg' in the tables. These two metrics about solution quality are presented together in one column 'min(avg)' for each algorithm.

- The averaged run time to identify the final vertex cover over the 10 executions, where the run time in an execution is the time to find the best found solution in that execution. The average run time is denoted by 'time' in the tables. In our experiments, the time is CPU time (measured in seconds), rather than wall clock time.

When comparing different algorithms, we put a higher priority on the solution quality than the run time, as in previous literatures for MinVC (Richter et al., 2007; Pullan, 2009; Cai et al., 2011, 2013) and the international algorithm competition for the NP-hard combinatorial optimization problems such as maximum satisfiability (Argelich, Li, Manyà, \& Planes, 2016). In detail, the rules of algorithm comparison and the reporting method are as follows:

1. For two algorithms A and B on an instance, we say algorithm A performs better than algorithm B w.r.t. solution quality, if and only if ('min' of $\mathrm{A}<$ 'min' of $\mathrm{B} \&$

2. http://www.graphrepository.com/networks.php, accessed on Jan. 2015. 
'avg' of $A \leq$ 'avg' of B) or ('min' of A $\leq$ 'min' of B \& 'avg' of A < 'avg' of B); we say algorithm $A$ and algorithm $B$ have the same performance w.r.t. solution quality if and only if ('min' of $\mathrm{A}=$ 'min' of $\mathrm{B} \&$ 'avg' of $\mathrm{A}=$ 'avg' of $\mathrm{B}$ ).

2. The algorithm that has the best performance w.r.t. solution quality is considered as the best algorithm for the instance. If more than one algorithms has the same solution quality which is better than other algorithms, then they are considered equally the best for the instance. The best 'min' and the best 'avg' values are indicated in bold face.

3. If for an instance, there exist two algorithms A and B that cannot be compared in terms of solution quality (according to principle 1), then we say there is not a clear dominant algorithm for that instance. In this case, the best 'min' and the best 'avg' values are also indicated in bold face, even though they are obtained by different algorithms.

4. Only when all algorithms obtain the same solution quality performance, we compare the run time of the algorithms, and the algorithm with the minimum value of average run time is the best algorithm, and its averaged time is indicated in bold face.

\section{A New Construction Algorithm for Vertex Cover}

This section investigates construction algorithms for MinVC. We first review previous construction algorithms, and then propose a new construction algorithm called EdgeGreedyVC. We compare different construction algorithms through both theoretical and experimental analysis.

\subsection{Previous Construction Algorithms for Vertex Cover}

Seen from the literature, there are two popular construction algorithms for vertex cover. The first one is based on finding a maximal matching, which has an approximation ratio of 2 (Papadimitrious $\&$ Steiglitz, 1982). The second one is a greedy algorithm that is employed in most practical MinVC algorithms.

\subsubsection{Maximal Matching based Construction Algorithm}

Given a graph $G=(V, E)$, a matching $M$ in $G$ is a set of pairwise non-adjacent edges, that is, no two edges share a common vertex. A maximal matching of a graph $G$ is a matching $M$ with the property that if any edge not in $M$ is added to $M$, it is no longer a matching, that is, $M$ is maximal if it is not a proper subset of any other matching in graph $G$.

A well-known construction algorithm for vertex cover is to find a maximal matching in the graph, and return the vertices in the matching as a vertex cover. Let us denote the vertex set of the found maximal matching $M$ as $V(M)$. It is easy to prove that $V(M)$ is a vertex cover. Suppose that there is an edge $e$ not covered by $V(M)$, then $e$ has no common vertex with all edges in $M$. Thus, we can extend matching $M$ by adding edge $e$, obtaining a greater sized matching $M^{\prime}=M \cup\{e\}$. This contradicts the fact that $M$ is a maximal matching.

For convenience, we denote this algorithm as MatchVC. For a graph $G=(V, E)$, beginning with an empty vertex set $C$, the MatchVC algorithm can be described as follows:

For each edge e $\in E$ : if e is not covered by $C$, add both endpoints of e into $C$. Return $C$.

It is obvious the complexity of the MatchVC algorithm is $O(m)$. This algorithm is very fast and guarantees an approximation ratio of 2 . Note that the best known approximation ratio is $2-o(1)$ 
(Karakostas, 2005), which is essentially not better than 2. However, the MatchVC algorithm does not return sufficiently good solutions in practice, which will also be shown in our experiments.

\subsubsection{Greedy Construction Algorithm}

Another construction algorithm for MinVC is an intuitive greedy procedure based on the gain values of vertices (Papadimitrious \& Steiglitz, 1982). It is the most commonly used construction algorithm for MinVC and is usually used to obtain the initial solution in local search algorithms for MinVC (Richter et al., 2007; Cai et al., 2011, 2013).

For convenience, we denote this algorithm as GreedyVC. For a graph $G=(V, E)$, beginning with an empty vertex set $C$, the GreedyVC algorithm works as follows:

Repeat the following operations until $C$ becomes a vertex cover: select a vertex $v \notin C$ with the maximum gain to add into $C$, breaking ties randomly. Return $C$.

The number of iterations of this procedure equals the size of the vertex cover $C$, and is denoted as $\ell$. We analyze the worst case complexity for two implementations of the above algorithm as follows. A straight-forward implementation is to scan the vertex set $V$ in each iteration in order to find the objective vertex, which has a complexity of $\Theta(n)$ for each iteration. Therefore, the complexity is $\Theta(\ell \cdot n)=O\left(n^{2}\right)$. A more "clever" implementation is to maintain the $C$ set and also a set of vertices not in $C$, which is denoted as $H$. In each iteration, we scan the $H$ set to find a vertex with the maximum gain. To be precise, we use $C_{i}$ and $H_{i}$ to denote the $C$ set and $H$ set at the beginning of the $i^{t h}$ iteration. We have $\left|C_{i}\right|=i-1$ and $\left|H_{i}\right|=n-\left|C_{i}\right|=n-(i-1)$. Thus, $\sum_{i=1}^{\ell}\left|H_{i}\right|=\sum_{i=1}^{\ell}(n-(i-1))=\frac{1}{2} \ell(2 n+1-\ell)$. Since $1 \leq \ell \leq n \Rightarrow \frac{1}{2} \ell(n+1) \leq \frac{1}{2} \ell(2 n+$ $1-\ell) \leq \ell n$, we have $\sum_{i=1}^{\ell}\left|H_{i}\right|=\Theta(\ell \cdot n)$. Therefore, the complexity of this implementation is $\sum_{i=1}^{\ell}\left|H_{i}\right|=\Theta(\ell \cdot n)=O\left(n^{2}\right)$.

We will see from the experiment results in Section 3.3 that, a quadratic complexity is too high for massive graphs and makes the algorithms inefficient so that they may fail to provide a vertex cover within a reasonable amount of time (like 1000 seconds). In Section 5, we re-implement this GreedyVC algorithm by using the heap data structure, which accelerates the procedure significantly, and we also improve GreedyVC by removing redundant vertices.

\subsection{The EdgeGreedyVC Algorithm}

We propose a fast vertex cover construction algorithm, which is called EdgeGreedyVC. The pseudo-code of EdgeGreedyVC is given in Algorithm 1. The EdgeGreedyVC Algorithm consists of an extending phase and a shrinking phase.

The extending phase: Starting with an empty set $C$, the algorithm extends $C$ by checking and covering an edge in each iteration. If the considered edge is uncovered, the endpoint with a higher degree is added into $C$. If the two endpoints have the same degree, we simply choose the first one. In this way, the algorithm is deterministic, since it does not utilize random choices. Obviously, we obtain a vertex cover at the end of the extending phase.

The shrinking phase: First, we calculate the loss values of vertices in $C$; then, we scan the $C$ set and if a vertex $v \in C$ has a loss value of 0 , it is removed, and loss values of its neighbors are updated accordingly.

Theorem 1. The EdgeGreedyVC procedure returns a minimal vertex cover in $O(m)$ time, where $m$ is the number of edges. 


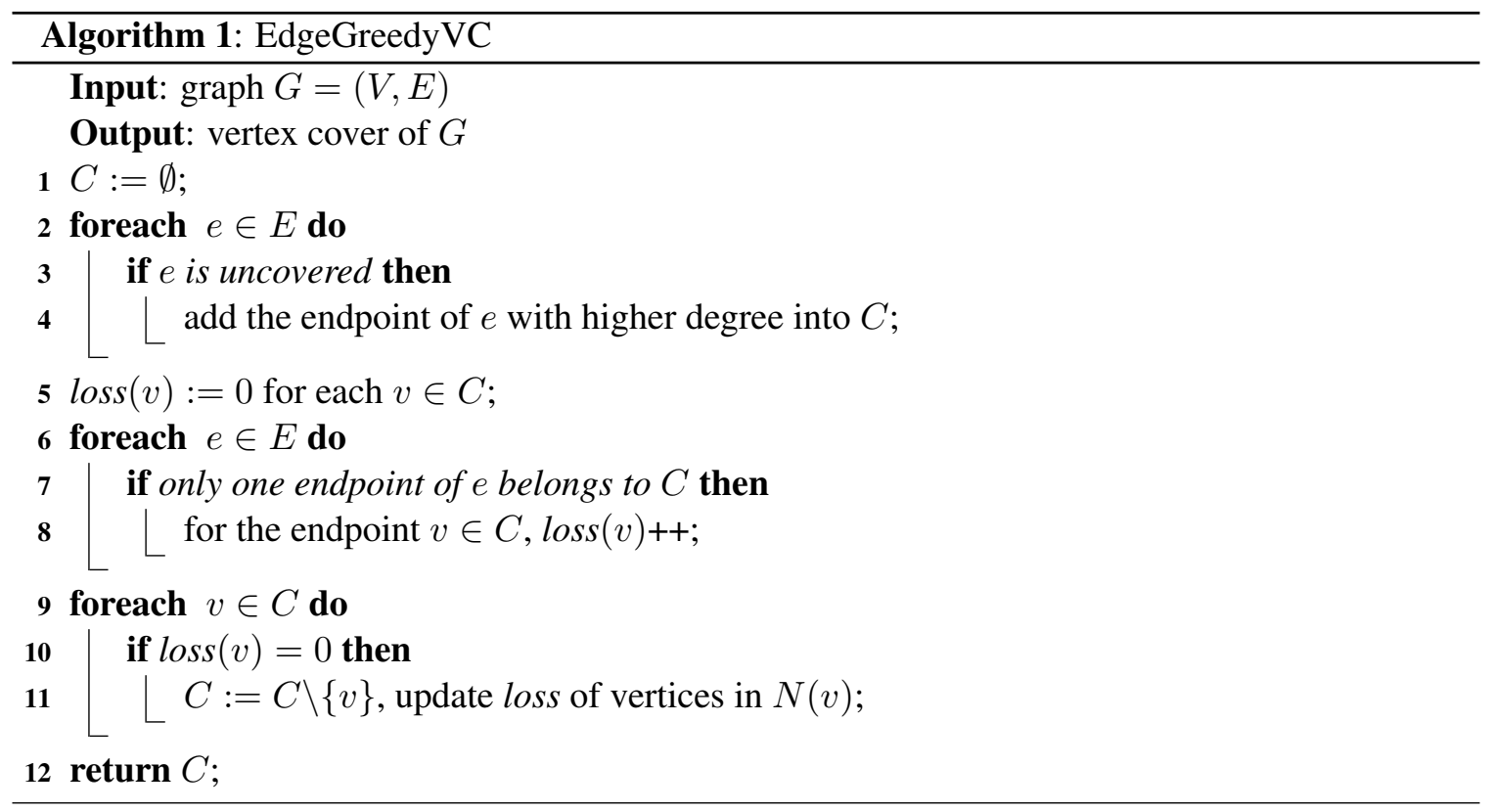

Proof: We first prove that the vertex set that the EdgeGreedyVC procedure returns is a minimal vertex cover. Let us use $C$ to denote the current vertex set during the procedure.

At the beginning of the shrinking phase, $C$ is a vertex cover. Also, each vertex removed in the shrinking phase has a loss value of 0 , and thus removing such vertices does not generate any uncovered edge. Hence, $C$ is a vertex cover after the shrinking phase. Now we prove that the vertex cover $C$ after the shrinking phase is minimal. Suppose after the shrinking phase, there exists a vertex in $C$ whose removal keeps $C$ a vertex cover. Without loss of generality, let this vertex be $v_{j}$, the one considered at the $j^{t h}$ iteration of the shrinking phase. From the assumption, we have $\operatorname{loss}\left(v_{j}\right)=0$ at the end of the shrinking phase. Notice that during the shrinking phase, the loss value of any vertex in $C$ does not decrease ${ }^{3}$. Thus, the value of $\operatorname{loss}\left(v_{j}\right)$ at the $j^{\text {th }}$ iteration is at most 0 , but loss values are non-negative, so it is 0 . Therefore, $v_{j}$ would have been removed at the $j^{\text {th }}$ iteration. This completes the proof by contradiction.

In the following, we calculate the time complexity of EdgeGreedyVC. The EdgeGreedyVC procedure can be divided into three parts: the first part (lines 2-4) performs the extending phase, the second part (lines 5-8) initializes the loss values, while the last one (lines 9-11) removes redundant vertices. Let $C^{+}$denotes the vertex cover obtained by the extending phase. It is clear that the complexity of the extending phase is $O(m)$. For the second part, the complexity is $O\left(\left|C^{+}\right|+m\right)$. Since at most one vertex is added in each iteration of the extending phase, we have $\left|C^{+}\right| \leq m$, and thus the complexity for the second part is $O(m)$. For the last part, the complexity depends on the total number of updating operations of loss values, which is calculated as $\sum_{v \in C^{+}} \operatorname{deg}(v)<$ $\sum_{v \in V} \operatorname{deg}(v)=2 m$. Therefore, the EdgeGreedyVC procedure has a complexity of $O(m)$.

Many massive real-world graphs are sparse graphs (Barabási \& Albert, 1999; Eubank, Kumar, Marathe, Srinivasan, \& Wang, 2004; Lu \& Chung, 2006), and heuristics with $O(m)$ complexity

3. This can be easily proved according to the definition of loss. 
are fast on such graphs. Nevertheless, we note that there are also dense graphs from real-world applications, and our method here is particularly effective for large sparse graphs.

\subsection{Comparing Construction Heuristics}

In this subsection, we carry out experiments to compare the three construction algorithms, namely MatchVC, GreedyVC and EdgeGreedyVC. We adopt the implementation of GreedyVC in NuMVC (2013), and we implement MatchVC using our codes of FastVC.

Since MatchVC and EdgeGreedyVC are deterministic algorithms, they are executed once (with random seed 1) on each instance. GreedyVC uses a randomized strategy to break ties, so it is executed 10 times (with random seeds from 1 to 10) on each instance and the averaged results over the 10 runs are reported. We report the size of vertex cover and the run time for each algorithm. The best solution size for each instance is presented in bold face. The experiment results are presented in Tables 1 and 2. We also report the size of the graphs in these two tables. The results can be summarized in the following observations.

1. The two greedy algorithms GreedyVC and EdgeGreedyVC always find better solutions than the MatchVC algorithm. GreedyVC and EdgeGreedyVC are competitive and complementary to each other. More specifically, GreedyVC finds the best solutions among all the three algorithms for 61 instances, and EdgeGreedyVC does this for 47 instances.

2. EdgeGreedyVC and MatchVC are much faster than GreedyVC. In particular, EdgeGreedyVC and MatchVC terminate within one second for all instances, while GreedyVC requires more than 100 seconds for 23 instances.

3. Overall, EdgeGreedyVC takes a very good balance between solution quality and run time, and it is a better choice when compared to GreedyVC and MatchVC.

\section{A New Local Search Algorithm for MinVC}

In this section, we propose a local search algorithm for MinVC called FastVC. We utilize the EdgeGreedyVC algorithm to construct the starting vertex cover for local search. Further, we propose a probabilistic method for choosing the vertex to remove in each step, which is an important idea in FastVC. Experiments are carried out to compare FastVC with the latest state of the art local search algorithm for MinVC namely NuMVC on massive graphs.

\subsection{The High Level Algorithm}

We first describe the FastVC algorithm from a high level. Details of important functions in FastVC and further analysis will be presented in the next subsection.

Local search algorithms for MinVC based on iteratively solving the decision problem start with a vertex cover, which we call the starting vertex cover. A small starting vertex cover can save the subsequent local search from too much unnecessary search before beginning seeking a good solution. A balance must be struck between the quality of the starting vertex cover and the time consumed in constructing it. Otherwise, the resulting algorithm may be inefficient in practice. FastVC uses the EdgeGreedyVC algorithm to construct the starting vertex cover. 
Table 1: Comparing three construction algorithms for MinVC.

\begin{tabular}{|c|c|c|c|c|c|c|c|c|}
\hline \multirow{2}{*}{ instance } & \multirow{2}{*}{$|\mathrm{V}|$} & \multirow{2}{*}{$|\mathrm{E}|$} & \multicolumn{2}{|c|}{ GreedyVC } & \multicolumn{2}{|c|}{ EdgeGreedyVC } & \multicolumn{2}{|c|}{ MatchVC } \\
\hline & & & avg size & time & size & time & size & time \\
\hline bio-celegans & 453 & 2025 & 258.3 & $<0.01$ & 259 & $<0.01$ & 398 & $<0.01$ \\
\hline bio-diseasome & 516 & 1188 & 285.2 & $<0.01$ & 285 & $<0.01$ & 400 & $<0.01$ \\
\hline bio-dmela & 7393 & 25569 & 2666.9 & 0.03 & 2717 & $<0.01$ & 4076 & $<0.01$ \\
\hline bio-yeast & 1458 & 1948 & 462.3 & $<0.01$ & 462 & $<0.01$ & 800 & $<0.01$ \\
\hline ca-AstroPh & 17903 & 196972 & 11517.8 & 0.6 & 11511 & $<0.01$ & 15376 & $<0.01$ \\
\hline ca-citeseer & 227320 & 814134 & 129356 & 88.2 & 129258 & $<0.01$ & 173614 & 0.01 \\
\hline ca-coauthors-dblp & 540486 & 15245729 & 472389.2 & 610.01 & 472259 & 0.04 & 510992 & 0.02 \\
\hline ca-CondMat & 21363 & 91286 & 12519.1 & 0.81 & 12497 & $<0.01$ & 17528 & $<0.01$ \\
\hline ca-CSphd & 1882 & 1740 & 555.3 & $<0.01$ & 553 & $<0.01$ & 1044 & $<0.01$ \\
\hline ca-dblp-2010 & 226413 & 716460 & 122194.3 & 84.37 & 122073 & $<0.01$ & 187206 & 0.01 \\
\hline ca-dblp-2012 & 317080 & 1049866 & 165268 & 168.36 & 165084 & 0.01 & 227224 & $<0.01$ \\
\hline ca-Erdos992 & 6100 & 7515 & 461 & $<0.01$ & 461 & $<0.01$ & 594 & $<0.01$ \\
\hline ca-GrQc & 4158 & 13422 & 2220 & 0.01 & 2214 & $<0.01$ & 3214 & $<0.01$ \\
\hline ca-HepPh & 11204 & 117619 & 6574.4 & 0.19 & 6565 & $<0.01$ & 9088 & $<0.01$ \\
\hline ca-hollywood-2009 & 1069126 & 56306653 & 864251.2 & 2182.47 & 864186 & 0.16 & 1040514 & 0.06 \\
\hline ca-MathSciNet & 332689 & 820644 & 140695.2 & 125.73 & 140446 & 0.01 & 203770 & $<0.01$ \\
\hline ca-netscience & 379 & 914 & 214 & $<0.01$ & 214 & $<0.01$ & 300 & $<0.01$ \\
\hline ia-email-EU & 32430 & 54397 & 820 & 0.02 & 827 & $<0.01$ & 1410 & $<0.01$ \\
\hline ia-email-univ & 1133 & 5451 & 609.8 & $<0.01$ & 615 & $<0.01$ & 814 & $<0.01$ \\
\hline ia-enron-large & 33696 & 180811 & 12825.3 & 1.04 & 12822 & $<0.01$ & 17504 & $<0.01$ \\
\hline ia-enron-only & 143 & 623 & 87.3 & $<0.01$ & 87 & $<0.01$ & 126 & $<0.01$ \\
\hline ia-fb-messages & 1266 & 6451 & 594.7 & $<0.01$ & 592 & $<0.01$ & 932 & $<0.01$ \\
\hline ia-infect-dublin & 410 & 2765 & 297.4 & $<0.01$ & 300 & $<0.01$ & 372 & $<0.01$ \\
\hline ia-infect-hyper & 113 & 2196 & 93 & $<0.01$ & 93 & $<0.01$ & 108 & $<0.01$ \\
\hline ia-reality & 6809 & 7680 & 81 & $<0.01$ & 81 & $<0.01$ & 114 & $<0.01$ \\
\hline ia-wiki-Talk & 92117 & 360767 & 17411.9 & 2.65 & 17464 & $<0.01$ & 26246 & $<0.01$ \\
\hline inf-power & 4941 & 6594 & 2277.8 & 0.01 & 2271 & $<0.01$ & 3736 & $<0.01$ \\
\hline inf-roadNet-CA & 1957027 & 2760388 & 1070458.5 & 7409.41 & 1062463 & 0.04 & 1660956 & 0.02 \\
\hline inf-roadNet-PA & 1087562 & 1541514 & 593581.3 & 2291.05 & 588269 & 0.03 & 915948 & 0.01 \\
\hline inf-road-usa & 23947347 & 28854312 & $\mathrm{n} / \mathrm{a}$ & $\mathrm{n} / \mathrm{a}$ & 12200485 & 0.7 & 19475384 & 0.23 \\
\hline rec-amazon & 91813 & 125704 & 49245.9 & 15.83 & 48542 & $<0.01$ & 74366 & $<0.01$ \\
\hline rt-retweet & 96 & 117 & 32.5 & $<0.01$ & 33 & $<0.01$ & 60 & $<0.01$ \\
\hline rt-retweet-crawl & 1112702 & 2278852 & 81572.8 & 115.24 & 82531 & 0.02 & 157810 & 0.01 \\
\hline rt-twitter-copen & 761 & 1029 & 238.1 & $<0.01$ & 238 & $<0.01$ & 422 & $<0.01$ \\
\hline socfb-A-anon & 3097165 & 23667394 & 376526.9 & 1577.91 & 424586 & 0.29 & 715962 & 0.05 \\
\hline socfb-B-anon & 2937612 & 20959854 & 303568.5 & 1129.9 & 342092 & 0.29 & 586904 & 0.04 \\
\hline socfb-Berkeley 13 & 22900 & 852419 & 17520.6 & 1.21 & 17599 & $<0.01$ & 21652 & 0.01 \\
\hline socfb-CMU & 6621 & 249959 & 5068 & 0.08 & 5090 & $<0.01$ & 6290 & $<0.01$ \\
\hline socfb-Duke14 & 9885 & 506437 & 7807.4 & 0.22 & 7838 & $<0.01$ & 9422 & $<0.01$ \\
\hline socfb-Indiana & 29732 & 1305757 & 23738.9 & 2.17 & 23788 & $<0.01$ & 28508 & $<0.01$ \\
\hline socfb-MIT & 6402 & 251230 & 4734.6 & 0.07 & 4756 & $<0.01$ & 6014 & $<0.01$ \\
\hline socfb-OR & 63392 & 816886 & 37269.7 & 6.09 & 37402 & $<0.01$ & 48342 & $<0.01$ \\
\hline socfb-Penn94 & 41536 & 1362220 & 31764.1 & 4.1 & 31851 & $<0.01$ & 39246 & 0.01 \\
\hline socfb-Stanford3 & 11586 & 568309 & 8634.9 & 0.28 & 8696 & $<0.01$ & 10796 & $<0.01$ \\
\hline socfb-Texas84 & 36364 & 1590651 & 28677.5 & 3.23 & 28762 & $<0.01$ & 34876 & 0.01 \\
\hline socfb-uci-uni & 58790782 & 92208195 & 866783 & 48316 & 869726 & 1 & 1732226 & 0.43 \\
\hline socfb-UCLA & 20453 & 747604 & 15500.7 & 0.94 & 15553 & $<0.01$ & 19226 & 0.01 \\
\hline socfb-UConn & 17206 & 604867 & 13474.9 & 0.67 & 13527 & $<0.01$ & 16466 & 0.01 \\
\hline socfb-UCSB37 & 14917 & 482215 & 11479.9 & 0.48 & 11510 & $<0.01$ & 14116 & $<0.01$ \\
\hline socfb-UF & 35111 & 1465654 & 27911.4 & 3.03 & 27894 & $<0.01$ & 33570 & 0.01 \\
\hline socfb-UIllinois & 30795 & 1264421 & 24532.3 & 2.31 & 24603 & $<0.01$ & 29512 & $<0.01$ \\
\hline socfb-Wisconsin 87 & 23831 & 835946 & 18725.9 & 1.33 & 18782 & $<0.01$ & 22750 & $<0.01$ \\
\hline
\end{tabular}


Table 2: Comparing three construction algorithms for MinVC (continued).

\begin{tabular}{|c|c|c|c|c|c|c|c|c|}
\hline \multirow{2}{*}{ instance } & \multirow{2}{*}{$|\mathrm{V}|$} & \multirow{2}{*}{$\mid \mathrm{El}$} & \multicolumn{2}{|c|}{ GreedyVC } & \multicolumn{2}{|c|}{ EdgeGreedyVC } & \multicolumn{2}{|c|}{ MatchVC } \\
\hline & & & avg size & avg time & size & time & size & time \\
\hline sc-ldoor & 952203 & 20770807 & 858313.1 & 1485.45 & 857967 & 0.06 & 893510 & 0.03 \\
\hline sc-msdoor & 415863 & 9378650 & 382176.7 & 270.99 & 382115 & 0.02 & 398824 & 0.02 \\
\hline sc-nasasrb & 54870 & 1311227 & 51714.1 & 5.04 & 51700 & $<0.01$ & 54870 & 0.01 \\
\hline sc-pkustk11 & 87804 & 2565054 & 84155.6 & 11.91 & 84355 & $<0.01$ & 87804 & $<0.01$ \\
\hline sc-pkustk13 & 94893 & 3260967 & 89714.2 & 15.23 & 89868 & $<0.01$ & 94534 & $<0.01$ \\
\hline sc-pwtk & 217891 & 5653221 & 208842.5 & 74.78 & 208255 & 0.01 & 217804 & $<0.01$ \\
\hline sc-shipsec1 & 140385 & 1707759 & 119977.5 & 39.69 & 120339 & $<0.01$ & 140354 & $<0.01$ \\
\hline sc-shipsec5 & 179104 & 2200076 & 149495.2 & 53.09 & 150957 & $<0.01$ & 179038 & $<0.01$ \\
\hline soc-BlogCatalog & 88784 & 2093195 & 20974 & 3.17 & 21257 & $<0.01$ & 29990 & 0.01 \\
\hline soc-brightkite & 56739 & 212945 & 21489.4 & 2.98 & 21469 & $<0.01$ & 31734 & $<0.01$ \\
\hline soc-buzznet & 101163 & 2763066 & 31074.4 & 6.6 & 31795 & 0.01 & 48174 & $<0.01$ \\
\hline soc-delicious & 536108 & 1365961 & 87670.5 & 68.91 & 90812 & 0.01 & 142016 & 0.01 \\
\hline soc-digg & 770799 & 5907132 & 104624.5 & 102.74 & 106831 & 0.03 & 133384 & 0.01 \\
\hline soc-dolphins & 62 & 159 & 34.6 & $<0.01$ & 35 & $<0.01$ & 50 & $<0.01$ \\
\hline soc-douban & 154908 & 327162 & 8718.6 & 1.36 & 8704 & $<0.01$ & 16156 & $<0.01$ \\
\hline soc-epinions & 26588 & 100120 & 9867.2 & 0.58 & 9860 & $<0.01$ & 14758 & $<0.01$ \\
\hline soc-flickr & 513969 & 3190452 & 154487.2 & 159.06 & 154471 & 0.02 & 225688 & 0.01 \\
\hline soc-flixster & 2523386 & 7918801 & 96498.4 & 216.44 & 96873 & 0.04 & 122862 & 0.01 \\
\hline soc-FourSquare & 639014 & 3214986 & 90688 & 64.71 & 90719 & 0.01 & 116474 & $<0.01$ \\
\hline soc-gowalla & 196591 & 950327 & 85443.7 & 45.3 & 85538 & 0.01 & 124018 & 0.01 \\
\hline soc-karate & 34 & 78 & 14.1 & $<0.01$ & 14 & $<0.01$ & 22 & $<0.01$ \\
\hline soc-lastfm & 1191805 & 4519330 & 79169.9 & 85.85 & 80205 & 0.03 & 99564 & 0.01 \\
\hline soc-livejournal & 4033137 & 27933062 & 1894963.6 & 19572.94 & 1898700 & 0.31 & 2591926 & 0.08 \\
\hline soc-LiveMocha & 104103 & 2193083 & 44176.5 & 11.61 & 45314 & 0.01 & 62782 & $<0.01$ \\
\hline soc-orkut & 2997166 & 106349209 & 2216036 & 18879 & 2228033 & 0.5 & 2706294 & 0.15 \\
\hline soc-pokec & 1632803 & 22301964 & 860947.8 & 3717.96 & 902600 & 0.19 & 1203086 & 0.04 \\
\hline soc-slashdot & 70068 & 358647 & 22637.3 & 3.56 & 22665 & $<0.01$ & 34056 & 0.01 \\
\hline soc-twitter-follows & 404719 & 713319 & 2323 & 0.8 & 2419 & $<0.01$ & 4644 & $<0.01$ \\
\hline soc-wiki-Vote & 889 & 2914 & 414.1 & $<0.01$ & 414 & $<0.01$ & 650 & $<0.01$ \\
\hline soc-youtube & 495957 & 1936748 & 148168.5 & 168.35 & 149089 & 0.02 & 240756 & $<0.01$ \\
\hline soc-youtube-snap & 1134890 & 2987624 & 279088.1 & 678.8 & 279389 & 0.03 & 456406 & 0.01 \\
\hline tech-as-caida2007 & 26475 & 53381 & 3692.7 & 0.15 & 3704 & $<0.01$ & 6648 & $<0.01$ \\
\hline tech-as-skitter & 1694616 & 11094209 & 529835.2 & 1883.76 & 553244 & 0.09 & 889676 & 0.03 \\
\hline tech-internet-as & 40164 & 85123 & $\mathbf{5 7 1 4 . 9}$ & 0.38 & 5766 & $<0.01$ & 10872 & $<0.01$ \\
\hline tech-p2p-gnutella & 62561 & 147878 & 15838.6 & 2.01 & 15759 & $<0.01$ & 28238 & $<0.01$ \\
\hline tech-RL-caida & 190914 & 607610 & 75681.1 & 35.72 & 77990 & $<0.01$ & 120890 & $<0.01$ \\
\hline tech-routers-rf & 2113 & 6632 & 805.5 & $<0.01$ & 803 & $<0.01$ & 1376 & $<0.01$ \\
\hline tech-WHOIS & 7476 & 56943 & 2297.9 & 0.03 & 2305 & $<0.01$ & 3380 & $<0.01$ \\
\hline web-arabic-2005 & 163598 & 1747269 & 115499.4 & 41.66 & 115256 & $<0.01$ & 138022 & 0.01 \\
\hline web-BerkStan & 12305 & 19500 & 5496.1 & 0.14 & 5565 & $<0.01$ & 8432 & $<0.01$ \\
\hline web-edu & 3031 & 6474 & 1591.2 & $<0.01$ & 1451 & $<0.01$ & 2818 & $<0.01$ \\
\hline web-google & 1299 & 2773 & 498.8 & $<0.01$ & 499 & $<0.01$ & 748 & $<0.01$ \\
\hline web-indochina-2004 & 11358 & 47606 & 7423.8 & 0.17 & 7367 & $<0.01$ & 9720 & $<0.01$ \\
\hline web-it-2004 & 509338 & 7178413 & 415772.1 & 542.39 & 415521 & 0.01 & 447464 & 0.02 \\
\hline web-polblogs & 643 & 2280 & 246 & $<0.01$ & 246 & $<0.01$ & 406 & $<0.01$ \\
\hline web-sk-2005 & 121422 & 334419 & 58529.1 & 17.77 & 58375 & $<0.01$ & 84604 & $<0.01$ \\
\hline web-spam & 4767 & 37375 & 2345.2 & 0.02 & 2352 & $<0.01$ & 3566 & $<0.01$ \\
\hline web-uk-2005 & 129632 & 11744049 & 127774 & 41.66 & 127774 & 0.02 & 128626 & 0.01 \\
\hline web-webbase-2001 & 16062 & 25593 & 2686.8 & 0.05 & 2674 & $<0.01$ & 4248 & $<0.01$ \\
\hline web-wikipedia2009 & 1864433 & 4507315 & 660288.4 & 3060.03 & 662296 & 0.07 & 1031900 & 0.02 \\
\hline
\end{tabular}




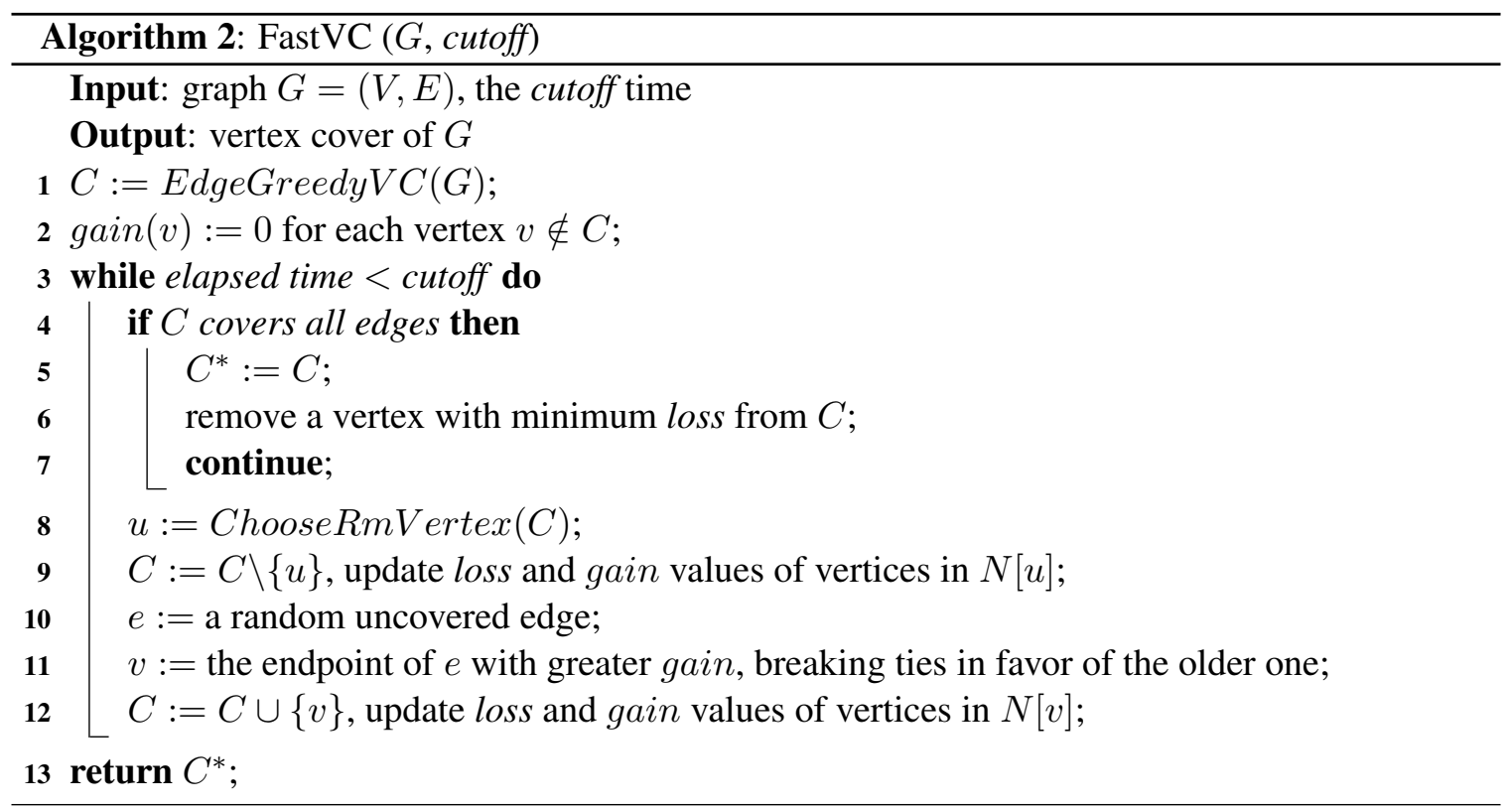

For the exchange step in local search, FastVC adopts the two-stage exchange framework, as it has lower complexity than the alternative paradigm based on vertex pair exchange. Indeed, thanks to the two-stage exchange framework, NuMVC performs several times more steps per second than other local search MinVC algorithms (Cai et al., 2013).

The FastVC algorithm is outlined in Algorithm 2, as described below. At the beginning, a vertex cover is constructed by the $E d g e G r e e d y V C$ function, which is taken as the initial candidate solution $C$ for the algorithm. The loss values of vertices in $C$ are calculated in the EdgeGreedyVC function. For vertices outside $C$, their gain values are set to 0 , as at this point all edges are covered by $C$ and adding any vertex into $C$ would not increase the number of covered edges.

Now we introduce the exchange step in FastVC. At each step, the algorithm first chooses a vertex in $u \in C$ to remove, which is accomplished by the ChooseRmVertex function. Then, the algorithm picks a random uncovered edge $e$, and chooses one of $e$ 's endpoints with the greater gain and adds it into $C$, breaking ties in favor of the older one. Note that along with removing or adding a vertex, the loss and gain values of the vertex and its neighbors are updated accordingly.

\subsection{Best from Multiple Selections (BMS)}

A critical function of FastVC is ChooseRmVertex, which returns a vertex from the candidate vertex set $C$ to remove in each exchange step. We propose a fast and effective heuristic for doing this task, which strikes a good balance between the time complexity and the quality of the selected vertex (w.r.t. the loss value).

Local search algorithms usually need to select an element from a candidate set. Perhaps the most commonly used strategy is to choose the best element according to some criterion, which we refer to as "best-picking" heuristic. With a suitable criterion, this heuristic guides the search towards the most promising area, and is thus widely adopted in local search algorithms. Recent examples of such heuristics for MinVC include the max-gain pair selection heuristic in COVER (Richter et al., 
2007) and the minimum loss removing heuristic in NuMVC (Cai et al., 2013). More examples can be found in local search algorithms for other famous NP-hard problems, such as the Satisfiability problem (Selman, Levesque, \& Mitchell, 1992; Hoos \& Stützle, 2004; Li \& Huang, 2005). Indeed, a lot of works on local search have been focused on the criterion for filtering the candidate set and the function for comparing elements, and once this is done, they simply pick the best one. The "best-picking" heuristic works well in most cases, but not for massive data sets where the candidate set is usually very large and finding the best element is very time-consuming.

We propose a cost-effective heuristic called Best from Multiple Selections (BMS), for picking a good element from a set. For a set $S$, the BMS heuristic works as follows:

Choose $k$ elements randomly with replacement from the set $S$, and then return the best one (w.r.t. some comparison function $f$ ), where $k$ is a parameter.

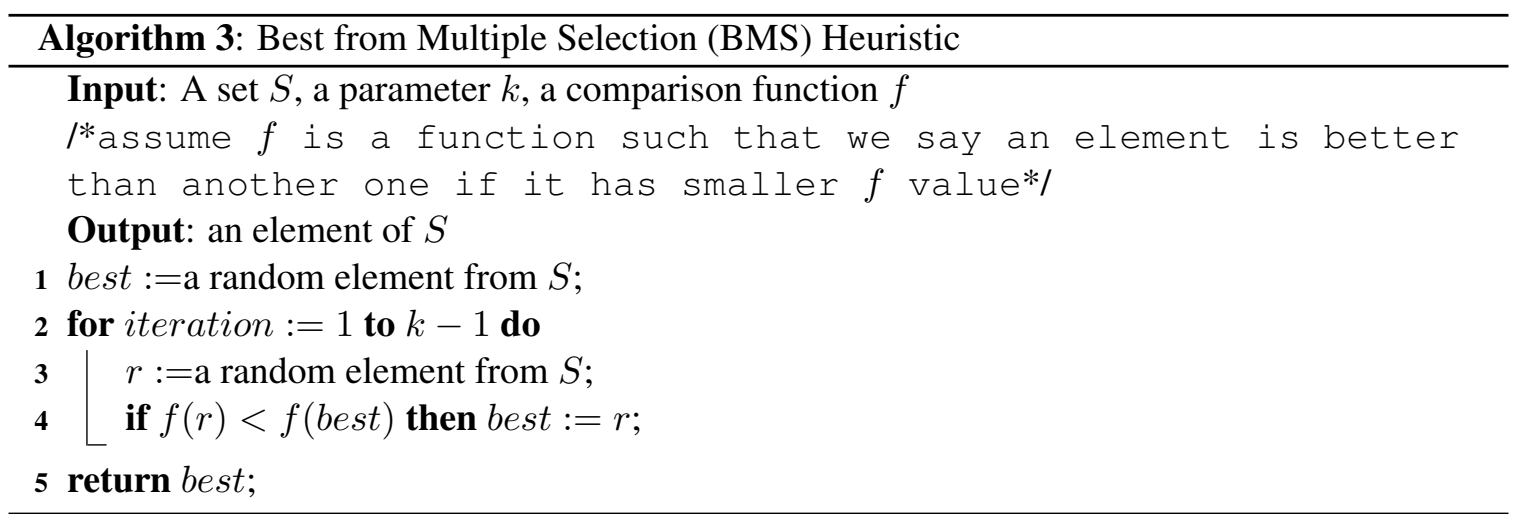

A more formal description of the BMS heuristic is given in Algorithm 3. Let us look at how well the BMS heuristic approximates the "best-picking" heuristic. For a real number $\rho \in(0,1)$, the probability of the event $E=\{$ the $f$ value of the element chosen by BMS is not greater than $\rho|S|$ elements in the set $S\}$ is $\operatorname{Pr}(E) \geq 1-\left(\frac{\rho|S|-1}{|S|}\right)^{k}>1-\rho^{k}$ (the " $\geq$ " is because there might be the case that more than one elements in those $\rho|S|$ elements have the same $f$ value, which is the minimum among $f$ values of all the $\rho|S|$ elements).

For ChooseRmVertex, the comparison function $f$ is simply the loss function on vertices, and we set $k=50$. Then, the probability that the BMS heuristic chooses a vertex whose loss value is not greater than $90 \%$ vertices in $C$ is $\operatorname{Pr}(E)>1-0.9^{50}>0.9948$. The above calculations illustrate that the BMS heuristic returns a vertex of good quality with a very high probability.

The complexity of the BMS heuristic is $O(k)=O(1)$, since $k$ is a constant. This is lower than $O(|C|)$ for the minimum loss heuristic used by previous local search algorithms for MinVC. Note that BMS is a generic heuristic and can be also applied to improve the time efficiency of local search algorithms for large scale instances of other problems.

\subsection{Experiments on FastVC}

We carry out experiments to evaluate FastVC on the real-world massive graphs, compared against the state of the art local search MinVC algorithm NuMVC. To illustrate the effectiveness of the local search procedure of FastVC, we also test a modified version of NuMVC (dubbed $\mathrm{NuMVC}_{e}$ ) which uses the EdgeGreedyVC construction heuristic with the same implementation as FastVC. 
The results show that FastVC significantly outperforms NuMVC and $\mathrm{NuMVC}_{e}$ on these massive graphs.

FastVC is built on the publicly available codes of NuMVC (2013) and uses the same data structure and the same implementation for the exchange step, yet it is simpler and lighter than NuMVC. Parameter settings of FastVC: For the BMS heuristic in the ChooseRmVertex function of FastVC, we set the $k$ parameter to 50, as mentioned in the previous section. This is based on preliminary experiments testing FastVC with different $k$ values. We test $k \in[10,100]$ with an increment step of 10 . When $k<10$ or $k>100$, the performance of the algorithm is obviously worse than that under $k \in[10,100]$. We observe that when $k \in[30,100]$, the performance is quite close, and FastVC with $k=50$ finds better solutions than the algorithm with $k=30,40$; also, FastVC with $k=50$ usually finds the same quality solutions as running the algorithm with $k>50$, but is usually faster.

For comparisons, we use the NuMVC algorithm (Cai et al., 2013) to represent the state of the art in solving the MinVC (and also MaxIS) problem. Based on experiments on DIMACS and BHOSLIB benchmarks, NuMVC is more reliably in finding the optimal or best known solution at speeds at least several times faster than earlier algorithms for MinVC and Maximum Independent Set (Cai et al., 2013). It is acknowledged as the latest breakthrough for MinVC solving in the literature (Fang, Chu, Qiao, Feng, \& Xu, 2014; Rosin, 2014; Jin \& Hao, 2015). Slightly better results have been reported for two algorithms built on the top of NuMVC (Fang et al., 2014; Cai, Lin, \& Su, 2015), but this does not materially change our conclusions below. The source code of NuMVC is online http://lcs.ios.ac.cn/ caisw/Code/NuMVC-Code.zip and also implemented in $\mathrm{C}++$. $\mathrm{NuMVC}_{e}$ is implemented in the source code of NuMVC by replacing the construction algorithm with the GreedyEdgeVC algorithm in FastVC.

The experiment comparing FastVC against NuMVC and $\mathrm{NuMVC}_{e}$ is conducted according to the experiment protocol in Section 2.3, and the results are reported in Tables 3 and 4. The results demonstrate that FastVC has better performance than NuMVC and $\mathrm{NuMVC}_{e}$. In detail, we have the following observations:

1. FastVC finds better vertex covers than NuMVC for 52 graphs and finds the same quality solutions for 40 graphs. FastVC finds worse solutions than NuMVC only for 10 graphs, and for 5 out of these 10 graphs, the two algorithms finds nearly the same quality solutions (with a gap of at most one vertex between the averaged sizes).

2. FastVC and NuMVC have similar performance on four classes of benchmarks, namely biological networks, interaction networks, Tweeter networks and technological networks. For other classes of benchmarks, FastVC significantly outperforms NuMVC.

3. Comparing their averaged run time, we found that FastVC is much faster than NuMVC on most of the graphs. In particular, for the 40 graphs where both algorithms find the same quality solutions, we compare the averaged time to obtain the final solution. FastVC is faster on 19 graphs, while NuMVC is faster on only 2 instances, and for the rest of the instances both algorithms have an averaged time of less than 0.01 seconds.

4. Although $\mathrm{NuMVC}_{e}$ shows improvement over NuMVC, particularly on those very large instances where NuMVC fails to provide a solution, the solutions returned by $\mathrm{NuMVC}_{e}$ are still worse than FastVC on most instances. 
Table 3: Comparing FastVC with NuMVC and $\mathrm{NuMVC}_{e}$, where $\mathrm{NuMVC}_{e}$ is modified from NuMVC that uses the same construction algorithm with the same implementation as FastVC.

\begin{tabular}{|c|c|c|c|c|c|c|}
\hline \multirow{2}{*}{ instance } & \multicolumn{2}{|l|}{ NuMVC } & \multicolumn{2}{|l|}{$\mathrm{NuMVC}_{e}$} & \multicolumn{2}{|l|}{ FastVC } \\
\hline & $\min (\operatorname{avg})$ & time & $\min (\operatorname{avg})$ & time & $\min (\operatorname{avg})$ & time \\
\hline bio-celegans & $249(249)$ & $<0.01$ & $249(249)$ & $<0.01$ & $249(249)$ & $<0.01$ \\
\hline bio-diseasome & $285(285)$ & $<0.01$ & $285(285)$ & $<0.01$ & $285(285)$ & $<0.01$ \\
\hline bio-dmela & $2630(2630)$ & 0.9 & $2630(2630)$ & 1.85 & $2630(2630)$ & $<0.01$ \\
\hline bio-yeast & $456(456)$ & $<0.01$ & $456(456)$ & $<0.01$ & $456(456)$ & $<0.01$ \\
\hline ca-AstroPh & $11483(11483)$ & 10.92 & $11483(11483)$ & 29.33 & $11483(11483)$ & 0.02 \\
\hline ca-citeseer & 129193(129195.5) & 153 & 129193(129193.6) & 59.58 & 129193(129193) & 1.07 \\
\hline ca-coauthors-dblp & $472251(472258.5)$ & 998.45 & $472180(472181.5)$ & 935.05 & $472179(472179)$ & 14.57 \\
\hline ca-CondMat & $12480(12480)$ & 46.59 & $12480(12480)$ & 36.97 & $12480(12480)$ & 0.02 \\
\hline ca-CSphd & $550(550)$ & $<0.01$ & $550(550)$ & $<0.01$ & $550(550)$ & $<0.01$ \\
\hline ca-dblp-2010 & 121971(121973.8) & 143.77 & 121972(121972.7) & 60.33 & $121969(121969)$ & 1.47 \\
\hline ca-dblp-2012 & 164952(164956.4) & 261.46 & 164951(164953.9) & 90.54 & 164949(164949) & 4.26 \\
\hline ca-Erdos992 & $461(461)$ & $<0.01$ & $461(461)$ & $<0.01$ & $461(461)$ & $<0.01$ \\
\hline ca-GrQc & $2208(2208)$ & 0.16 & $2208(2208)$ & $<0.01$ & $2208(2208)$ & $<0.01$ \\
\hline ca-HepPh & $6555(6555)$ & 18.9 & $6555(6555)$ & 6.59 & $6555(6555)$ & $<\mathbf{0 . 0 1}$ \\
\hline ca-hollywood-2009 & $\mathrm{n} / \mathrm{a}$ & $\mathrm{n} / \mathrm{a}$ & $864115(864115.4)$ & 990.79 & $864052(864052)$ & 25.59 \\
\hline ca-MathSciNet & 139982(139989.7) & 174.14 & 139982(139985.2) & 43.44 & 139951(139951) & 4.36 \\
\hline ca-netscience & $214(214)$ & $<0.01$ & $214(214)$ & $<0.01$ & $214(214)$ & $<0.01$ \\
\hline ia-email-EU & $820(820)$ & 0.02 & $820(820)$ & $<0.01$ & $820(820)$ & $<0.01$ \\
\hline ia-email-univ & $594(594)$ & $<0.01$ & $594(594)$ & $<0.01$ & $594(594)$ & $<0.01$ \\
\hline ia-enron-large & 12781(12781) & 52.47 & 12781(12781) & 121.21 & 12781(12781) & 0.04 \\
\hline ia-enron-only & $86(86)$ & $<0.01$ & $86(86)$ & $<0.01$ & $86(86)$ & $<0.01$ \\
\hline ia-fb-messages & $578(578)$ & $<0.01$ & $578(578)$ & $<0.01$ & $578(578)$ & $<0.01$ \\
\hline ia-infect-dublin & 293(293) & $<0.01$ & 293(293) & $<0.01$ & 293(293.2) & 480.11 \\
\hline ia-infect-hyper & $90(90)$ & $<0.01$ & $90(90)$ & $<0.01$ & $90(90)$ & $<0.01$ \\
\hline ia-reality & $81(81)$ & $<0.01$ & $81(81)$ & $<0.01$ & $81(81)$ & $<0.01$ \\
\hline ia-wiki-Talk & $17288(17288.2)$ & 510.58 & $17288(17288.4)$ & 704.41 & 17288(17288) & 0.08 \\
\hline inf-power & $2203(2203)$ & 1.05 & $2203(2203)$ & 1.54 & $2203(2203)$ & $<\mathbf{0 . 0 1}$ \\
\hline inf-roadNet-CA & $\mathrm{n} / \mathrm{a}$ & $\mathrm{n} / \mathrm{a}$ & 1044047(1044195.7) & 1000 & $1001273(1001311.2)$ & 896.33 \\
\hline inf-roadNet-PA & $\mathrm{n} / \mathrm{a}$ & $\mathrm{n} / \mathrm{a}$ & $560365(560410.8)$ & 947.85 & $555220(555243)$ & 652.14 \\
\hline inf-road-usa & $\mathrm{n} / \mathrm{a}$ & $\mathrm{n} / \mathrm{a}$ & 12198972(12198983.8) & 1000 & $12049567(12050440.1)$ & 1000 \\
\hline rec-amazon & $47655(47672.6)$ & 953.23 & $47671(47681.9)$ & 943.31 & $47606(47606)$ & 0.89 \\
\hline rt-retweet & $32(32)$ & $<0.01$ & $32(32)$ & $<0.01$ & $32(32)$ & $<0.01$ \\
\hline rt-retweet-crawl & $81043(81047.2)$ & 119.17 & $81057(81059.7)$ & 7.637 & $81048(81048)$ & 1.34 \\
\hline rt-twitter-copen & $237(237)$ & $<0.01$ & $237(237)$ & $<0.01$ & $237(237)$ & $<0.01$ \\
\hline socfb-A-anon & $\mathrm{n} / \mathrm{a}$ & $\mathrm{n} / \mathrm{a}$ & $375234(375236.2)$ & 677.28 & 375231(375232.8) & 128.55 \\
\hline socfb-B-anon & $\mathrm{n} / \mathrm{a}$ & $\mathrm{n} / \mathrm{a}$ & $303049(303050)$ & 466.86 & $303048(303048.9)$ & 85.1 \\
\hline socfb-Berkeley 13 & $17216(17218)$ & 515.58 & $17215(17218.1)$ & 517.30 & $17210(17212.7)$ & 290.4 \\
\hline socfb-CMU & 4986(4986.1) & 248.73 & 4986(4986.1) & 158.95 & 4986(4986.5) & 3.91 \\
\hline socfb-Duke14 & 7683(7683.6) & 212.95 & 7683(7683.6) & 149.95 & $7683(7683)$ & 228.3 \\
\hline socfb-Indiana & $23320(23326.5)$ & 556.5 & $23322(23327.9)$ & 455.31 & $23315(23317.1)$ & 517.27 \\
\hline socfb-MIT & 4657(4657) & 8.2 & 4657(4657) & 10.06 & $4657(4657)$ & 41.13 \\
\hline socfb-OR & $36558(36560.6)$ & 564.13 & $36557(36560.6)$ & 678.19 & $36548(36549.2)$ & 144.06 \\
\hline socfb-Penn94 & $31179(31183.3)$ & 559.12 & $31178(31181.6)$ & 632.33 & $31162(31164.8)$ & 552.92 \\
\hline socfb-Stanford3 & $8518(8518)$ & 8.77 & $8518(8518)$ & 8.84 & 8517(8517.9) & 101 \\
\hline socfb-Texas84 & $28174(28180.5)$ & 639.12 & 28176(28184.2) & 636.72 & 28167(28171.1) & 495.91 \\
\hline socfb-uci-uni & $\mathrm{n} / \mathrm{a}$ & $\mathrm{n} / \mathrm{a}$ & $866768(866768)$ & 488.22 & $866768(866768)$ & 37.05 \\
\hline socfb-UCLA & $15225(15227.1)$ & 399.21 & $15225(15227.4)$ & 489.63 & $15223(15224.3)$ & 297.92 \\
\hline socfb-UConn & 13231(13233) & 611.89 & 13231(13233.2) & 565.24 & $13230(13231.5)$ & 304.11 \\
\hline socfb-UCSB37 & $11262(11263)$ & 381.45 & $11262(\mathbf{1 1 2 6 2 . 8})$ & 367.34 & 11261(11263) & 210.62 \\
\hline socfb-UF & $27319(27323.5)$ & 607.36 & $27319(27323.5)$ & 400.43 & 27306(27309) & 459.23 \\
\hline socfb-UIllinois & $24096(24106.4)$ & 545.24 & $24100(24106.7)$ & 534.74 & $24091(24092.2)$ & 477.69 \\
\hline socfb-Wisconsin 87 & 18388(18390.6) & 733.22 & $18390(18392.2)$ & 505.07 & $18383(18385.1)$ & 295.36 \\
\hline
\end{tabular}

\section{The NuMVC2 and FastVC2 Algorithms}

We observe that the construction algorithms GreedyVC and MatchVC can be improved by applying the shrinking phase of EdgeGreedyVC after construction of the vertex cover is finished. Also, 
Table 4: Comparing FastVC with NuMVC and $\mathrm{NuMVC}_{e}$ (continued).

\begin{tabular}{|c|c|c|c|c|c|c|}
\hline \multirow{2}{*}{ instance } & \multicolumn{2}{|l|}{ NuMVC } & \multicolumn{2}{|l|}{$\mathrm{NuMVC}_{e}$} & \multicolumn{2}{|l|}{ FastVC } \\
\hline & $\min (\operatorname{avg})$ & time & $\min (\operatorname{avg})$ & time & $\min (\operatorname{avg})$ & time \\
\hline sc-ldoor & $\mathrm{n} / \mathrm{a}$ & $\mathrm{n} / \mathrm{a}$ & $856920(856930)$ & 997.46 & $856755(856757.4)$ & 218.94 \\
\hline sc-msdoor & $381569(381574.6)$ & 986.61 & $381564(381565.6)$ & 973.41 & $381558(381558.9)$ & 18.71 \\
\hline sc-nasasrb & $51244(\mathbf{5 1 2 4 6 . 7})$ & 781.64 & $51245(51247.2)$ & 781.34 & $51244(51247.3)$ & 517.13 \\
\hline sc-pkustk11 & $83911(\mathbf{8 3 9 1 1})$ & 539.67 & 83911(83911) & 571.60 & $83911(83912.5)$ & 6.84 \\
\hline sc-pkustk13 & $89218(89222)$ & 848.29 & $89219(89222)$ & 913.69 & $89217(89220.6)$ & 315.23 \\
\hline sc-pwtk & $207749(207756.3)$ & 249.46 & 207741(207746.1) & 147.74 & 207716(207719.9) & 184.89 \\
\hline sc-shipsec1 & $117477(117536.9)$ & 998.33 & $117468(117523.7)$ & 995.95 & $117318(117337.5)$ & 722.4 \\
\hline sc-shipsec5 & $147288(147324.5)$ & 990.71 & $147326(147339.9)$ & 991.17 & 147137(147173.8) & 569.7 \\
\hline soc-BlogCatalog & $20752(\mathbf{2 0 7 5 2})$ & 394.43 & $20752(20752.2)$ & 516.62 & 20752(20752) & 0.19 \\
\hline soc-brightkite & 21192(21193.5) & 841.9 & 21191(21192.8) & 890.157 & $21190(21190)$ & 0.2 \\
\hline soc-buzznet & 30613(30613.2) & 657.14 & $\mathbf{3 0 6 1 3 ( 3 0 6 1 3 . 9 )}$ & 648.62 & $30625(30625)$ & 15.95 \\
\hline soc-delicious & 85522(85585.6) & 78.09 & $85696(85715.2)$ & 947.90 & $85686(85696.4)$ & 2.54 \\
\hline soc-digg & $103303(103318.7)$ & 111.21 & 103371(103387) & 866.31 & $103244(103245.3)$ & 3.34 \\
\hline soc-dolphins & $34(34)$ & $<0.01$ & $34(34)$ & $<0.01$ & $34(34)$ & $<0.01$ \\
\hline soc-douban & $8685(8685)$ & 1.37 & $8685(8685)$ & 0.01 & $8685(8685)$ & $<0.01$ \\
\hline soc-epinions & $9757(9757)$ & 82.8 & $9757(9757)$ & 142.78 & $9757(9757)$ & 0.13 \\
\hline soc-flickr & $153343(153352.7)$ & 194.34 & $153340(153347.4)$ & 39.028 & 153272(153272) & 18.51 \\
\hline soc-flixster & 96319(96320.7) & 217.78 & 96321(96322.7) & 446.88 & 96317(96317) & 1.61 \\
\hline soc-FourSquare & 90125(90134.1) & 835.65 & 90127(90132) & 795.37 & 90108(90109.2) & 124.9 \\
\hline soc-gowalla & $84313(84322.6)$ & 940.31 & $84316(84324.5)$ & 919.63 & $84222(84222.3)$ & 64.58 \\
\hline soc-karate & $14(14)$ & $<0.01$ & $14(14)$ & $<0.01$ & $14(14)$ & $<0.01$ \\
\hline soc-lastfm & $78692(78695)$ & 87.43 & $78696(78698.2)$ & 817.99 & 78688(78688) & 0.9 \\
\hline soc-livejournal & $\mathrm{n} / \mathrm{a}$ & $\mathrm{n} / \mathrm{a}$ & 1888661(1888673.1) & 1000 & $1869046(1869051.1)$ & 953.11 \\
\hline soc-LiveMocha & $43430(43432.8)$ & 763.05 & $43432(43434.7)$ & 803.47 & $43427(43427)$ & 17.44 \\
\hline soc-orkut & $\mathrm{n} / \mathrm{a}$ & $\mathrm{n} / \mathrm{a}$ & $2205976(2206173.6)$ & 1000 & $2171296(2171380.5)$ & 996.66 \\
\hline soc-pokec & $\mathrm{n} / \mathrm{a}$ & $\mathrm{n} / \mathrm{a}$ & 847939(848147.4) & 1000 & 843422(843434.9) & 772.77 \\
\hline soc-slashdot & $\mathbf{2 2 3 7 3}(22377)$ & 774.65 & $22374(22376.5)$ & 848.95 & $22373(22373)$ & 0.2 \\
\hline soc-twitter-follows & $2323(2323)$ & 0.8 & $2323(2323)$ & 0.09 & $2323(2323)$ & 0.01 \\
\hline soc-wiki-Vote & $406(406)$ & $<0.01$ & $406(406)$ & $<0.01$ & $406(406)$ & $<0.01$ \\
\hline soc-youtube & $146456(146468.2)$ & 213.06 & $146464(146475.2)$ & 47.76 & $146376(146376)$ & 5.67 \\
\hline soc-youtube-snap & $277015(277025.2)$ & 889.32 & $277005(277016.8)$ & 203.18 & 276945(276945) & 12.74 \\
\hline tech-as-caida2007 & 3683(3683) & 2.32 & $3683(3683)$ & 2.88 & $3683(3683)$ & $<0.01$ \\
\hline tech-as-skitter & $\mathrm{n} / \mathrm{a}$ & $\mathrm{n} / \mathrm{a}$ & $527857(527881.2)$ & 818.69 & 527185(527195.9) & 446.49 \\
\hline tech-internet-as & $5700(5700)$ & 8.97 & $5700(5700)$ & 33.23 & $5700(5700)$ & 0.01 \\
\hline tech-p2p-gnutella & 15682(15682) & 24.25 & 15682(15682) & 43.68 & 15682(15682) & 0.01 \\
\hline tech-RL-caida & 74759(74776.5) & 979.74 & 75001(75015.7) & 991.45 & 74930(74938.9) & 9.62 \\
\hline tech-routers-rf & $795(795)$ & 0.01 & $795(795)$ & $<0.01$ & $795(795)$ & $<0.01$ \\
\hline tech-WHOIS & $2284(2284)$ & 0.31 & $2284(2284)$ & 0.55 & $2284(2284)$ & $<\mathbf{0 . 0 1}$ \\
\hline web-arabic-2005 & $114464(114471.8)$ & 111.51 & $114454(114458.6)$ & 64.80 & $114426(114427.2)$ & 323.58 \\
\hline web-BerkStan & $5384(5384)$ & 10 & $5384(5384)$ & 10.03 & $5384(5384)$ & 25.8 \\
\hline web-edu & $1451(1451)$ & 0.35 & $1451(1451)$ & $<\mathbf{0 . 0 1}$ & $1451(1451)$ & $<0.01$ \\
\hline web-google & $498(498)$ & $<0.01$ & $498(498)$ & $<0.01$ & $498(498)$ & $<0.01$ \\
\hline web-indochina-2004 & $7300(7300)$ & 14.54 & $7300(7300)$ & 13.876 & 7300(7300) & 0.09 \\
\hline web-it-2004 & $414741(414758.7)$ & 987.89 & $414675(414680.8)$ & 580.91 & $414671(414676.3)$ & 9.92 \\
\hline web-polblogs & $244(244)$ & $<0.01$ & $244(244)$ & $<0.01$ & $244(244)$ & $<0.01$ \\
\hline web-sk-2005 & $58199(58205.5)$ & 304.68 & $58205(58209.5)$ & 732.55 & $\mathbf{5 8 1 7 3}(58173)$ & 8.67 \\
\hline web-spam & 2297(2297) & 0.28 & 2297(2297) & 0.42 & $2298(2298)$ & $<0.01$ \\
\hline web-uk-2005 & 127774(127774) & 41.67 & 127774(127774) & 0.03 & 127774(127774) & 0.02 \\
\hline web-webbase-2001 & 2651(2651.9) & 48.54 & $2652(2652)$ & 1.36 & $2652(2652)$ & 0.01 \\
\hline web-wikipedia2009 & $\mathrm{n} / \mathrm{a}$ & $\mathrm{n} / \mathrm{a}$ & 649546(649571.6) & 999.78 & $648317(648321.8)$ & 692.3 \\
\hline
\end{tabular}

the construction algorithm GreedyVC is implemented in a way with quadratic complexity in the original implementation of NuMVC. In this section, we use a heap data structure to re-implement GreedyVC in NuMVC, which leads to a significant speedup. The resulting, improved algorithms based on GreedyVC and MatchVC are dubbed GreedyVC+ and MatchVC+ respectively. 
Table 5: Comparing EdgeGreedyVC, GreedyVC+ and MatchVC+, where '\#win.' counts the number of winning instances and accumulated time includes the run time on all 102 instances.

\begin{tabular}{llllll}
\hline & GreedyVC+ & & EdgeGreedyVC & & MatchVC+ \\
\cline { 2 - 2 } \cline { 5 - 6 } \#win. & 70 & & 25 & \\
Accumulated Time & 146.14 & & 6.26 & 6.35 \\
\hline
\end{tabular}

We conduct experiments to compare EdgeGreedyVC, GreedyVC+ and MatchVC+. Since EdgeGreedyVC and MatchVC+ are deterministic algorithms, they are executed once (with random seed 1) on each instance. GreedyVC+ uses a randomized strategy to break ties, so it is executed 10 times (with random seeds from 1 to 10) on each instance and the averaged results over the 10 runs are reported. Interestingly, our experiments show that these three construction MinVC algorithms have superiority on different instances (Table 5), where an algorithm wins an instance if it is the only one that gives the best solution or it has the least run time among the algorithms with the best quality solution. Therefore, a natural idea for improving local search MinVC algorithms is to integrate the three construction algorithms by using them to generate three solutions and take the best one as the initial solution for local search. We use this engineering practice to improve both NuMVC and FastVC, resulting in two local search MinVC solvers named NuMVC2 and FastVC2. They are also tested on the whole benchmark suite and the results are reported in Tables 6 and 7, which show that FastVC2 outperforms NuMVC2 on most instances. Since the initial solution is the same, this indicates the effectiveness of the local search procedure in FastVC for solving massive graphs.

Additionally, comparing the results in Tables 6 and 7 with those in Tables 3 and 4, we observe that NuMVC2 has better performance overall than NuMVC and $\mathrm{NuMVC}_{e}$, and FastVC2 is better than FastVC. Note that these algorithms use the same random seeds $(1,2, \ldots, 10)$ in our experiments. With respect to solution quality, NuMVC2 performs better than NuMVC on 31 instances while worse on 19 instances, and better than $\mathrm{NuMVC}_{e}$ on 28 instances while worse on 21 instances. FastVC2 performs better than FastVC on 26 instances while worse on 17 instances. We also test different variants of NuMVC and FastVC with only one construction heuristic from EdgeGreedyVC, GreedyVC+ and MatchVC+, and the results show that NuMVC2 and FastVC2 have overall better performance w.r.t. solution quality than their variants with a single construction heuristic.

\section{Improving MinVC Solving by Preprocessing Techniques}

In this section, we develop a preprocessing algorithm to simplify graphs for MinVC algorithms. The preprocessing algorithm works in two phases and uses four reduction rules. We conduct experiments to study the rules and show their effectiveness. The preprocessing algorithm is used to improve NuMVC2 and FastVC2 from the previous section, resulting in two MinVC solvers named NuMVC2+p and FastVC2+p. Experiments show that the preprocessing algorithm can improve the solution quality for a considerable portion of the tested graphs.

\subsection{Reduction Rules and the Preprocessing Algorithm}

Our preprocessing algorithm for MinVC is based on four reduction rules. The first three rules are very simple and have been widely used (Chen, Kanj, \& Jia, 2001; Abu-Khzam, Collins, Fellows, 
Table 6: Experiment results of NuMVC2 and FastVC2. Both algorithms use EdgeGreedyVC, GreedyVC+ and MatchVC+ to generate three solutions and take the best one as the initial solution for local search.

\begin{tabular}{|c|c|c|c|c|}
\hline \multirow{2}{*}{ instance } & \multicolumn{2}{|l|}{ NuMVC2 } & \multicolumn{2}{|l|}{ FastVC2 } \\
\hline & $\min (\operatorname{avg})$ & time & $\min (\operatorname{avg})$ & time \\
\hline bio-celegans & $249(249)$ & $<0.01$ & $249(249)$ & $<0.01$ \\
\hline bio-diseasome & $285(285)$ & $<0.01$ & $285(285)$ & $<0.01$ \\
\hline bio-dmela & $2630(2630)$ & 1.29 & $2632(2632)$ & 0.01 \\
\hline bio-yeast & $456(456)$ & $<0.01$ & $456(456)$ & $<0.01$ \\
\hline ca-AstroPh & $11483(11483)$ & 63.2 & $11483(11483)$ & 0.06 \\
\hline ca-citeseer & 129193(129193.6) & 60.63 & 129193(129193) & 1.36 \\
\hline ca-coauthors-dblp & $472180(472181.8)$ & 905.45 & 472179(472179) & 16.91 \\
\hline ca-CondMat & $12480(12480)$ & 39.03 & $12480(12480)$ & 0.04 \\
\hline ca-CSphd & $550(550)$ & $<0.01$ & $550(550)$ & $<0.01$ \\
\hline ca-dblp-2010 & $121972(121972.7)$ & 60.82 & $121969(121969)$ & 1.78 \\
\hline ca-dblp-2012 & 164951(164953.9) & 92.56 & $164949(164949)$ & 4.76 \\
\hline ca-Erdos992 & $461(461)$ & $<0.01$ & $461(461)$ & $<0.01$ \\
\hline ca-GrQc & $2208(2208)$ & $<0.01$ & $2208(2208)$ & $<0.01$ \\
\hline ca-HepPh & $6555(6555)$ & 6.48 & $6555(6555)$ & $\mathbf{0 . 0 3}$ \\
\hline ca-hollywood-2009 & $864116(864117.2)$ & 973.41 & $864052(864052)$ & 33.17 \\
\hline ca-MathSciNet & 139982(139985.2) & 45.07 & 139951(139951) & 4.54 \\
\hline ca-netscience & $214(214)$ & $<0.01$ & $214(214)$ & $<0.01$ \\
\hline ia-email-EU & $820(820)$ & $<0.01$ & $820(820)$ & $<0.01$ \\
\hline ia-email-univ & $594(594)$ & $<0.01$ & $594(594)$ & $<0.01$ \\
\hline ia-enron-large & $12781(12781)$ & 74.25 & $12781(12781)$ & 0.11 \\
\hline ia-enron-only & $86(86)$ & $<0.01$ & $86(86)$ & $<0.01$ \\
\hline ia-fb-messages & $578(578)$ & $<0.01$ & $578(578)$ & $<0.01$ \\
\hline ia-infect-dublin & 293(293) & $<0.01$ & 293(293) & $<0.01$ \\
\hline ia-infect-hyper & $90(90)$ & $<0.01$ & $90(90)$ & $<0.01$ \\
\hline ia-reality & $81(81)$ & $<0.01$ & $81(81)$ & $<0.01$ \\
\hline ia-wiki-Talk & $17288(17288.3)$ & 523.41 & $17288(\mathbf{1 7 2 8 8})$ & 0.14 \\
\hline inf-power & $2203(2203)$ & 1.7 & $2203(2203)$ & $<\mathbf{0 . 0 1}$ \\
\hline inf-roadNet-CA & $1038328(1038553)$ & 1000 & $1001442(1001474.1)$ & 893.82 \\
\hline inf-roadNet-PA & $560133(560173.4)$ & 936.31 & $555269(555324.6)$ & 700.32 \\
\hline inf-road-usa & $12082301(12082356.4)$ & 1000 & $11809276(11828598)$ & 1000 \\
\hline rec-amazon & $47671(47682.2)$ & 923.45 & $47606(47606)$ & 1.12 \\
\hline rt-retweet & $32(32)$ & $<0.01$ & $32(32)$ & $<0.01$ \\
\hline rt-retweet-crawl & $81044(81045.3)$ & 2.85 & $81040(81040)$ & 1.89 \\
\hline rt-twitter-copen & $237(237)$ & $<0.01$ & $237(237)$ & $<0.01$ \\
\hline socfb-A-anon & $375232(375233.4)$ & 39.19 & $375230(375230.9)$ & 23.7 \\
\hline socfb-B-anon & $303048(303049.5)$ & 26.39 & $303048(\mathbf{3 0 3 0 4 8})$ & 7.15 \\
\hline socfb-Berkeley 13 & $17213(17216.8)$ & 579.99 & $17210(17211.2)$ & 345.5 \\
\hline socfb-CMU & 4986(4986.1) & 193.77 & 4987(4987) & 3.49 \\
\hline socfb-Duke14 & $7683(7683.5)$ & 115.93 & $7683(\mathbf{7 6 8 3})$ & 361.41 \\
\hline socfb-Indiana & $23323(23328.8)$ & 503.05 & $23315(23317.1)$ & 407.46 \\
\hline socfb-MIT & $4657(4657)$ & 7.97 & $4657(4657)$ & 21.47 \\
\hline socfb-OR & $36559(36562.6)$ & 663.33 & $36548(36548.7)$ & 288.97 \\
\hline socfb-Penn94 & $31177(31185.1)$ & 484.42 & $31163(31165.2)$ & 600.93 \\
\hline socfb-Stanford3 & $8518(8518)$ & 10.74 & $8518(8518)$ & 25.22 \\
\hline socfb-Texas84 & $28180(28184.3)$ & 613.51 & 28165(28168.7) & 629.11 \\
\hline socfb-uci-uni & $866766(866766)$ & 127.19 & $866766(866766)$ & 26.07 \\
\hline socfb-UCLA & $15225(15227.6)$ & 656.43 & $15224(15225.3)$ & 355.59 \\
\hline socfb-UConn & $13231(13233.6)$ & 589.34 & $13230(13230.9)$ & 352.79 \\
\hline socfb-UCSB37 & $11262(11263)$ & 546.05 & $11261(11262.5)$ & 269.29 \\
\hline socfb-UF & $27322(27325.3)$ & 544.41 & $27306(27308.6)$ & 348.77 \\
\hline socfb-UIllinois & 24103(24108.2) & 561.88 & $24093(24095.2)$ & 373.81 \\
\hline socfb-Wisconsin 87 & 18389(18392.1) & 636.37 & 18383(18385) & 569.64 \\
\hline
\end{tabular}


Table 7: Experiment results of NuMVC2 and FastVC2 (continued).

\begin{tabular}{|c|c|c|c|c|}
\hline \multirow{2}{*}{ instance } & \multicolumn{2}{|l|}{ NuMVC2 } & \multicolumn{2}{|l|}{ FastVC2 } \\
\hline & $\min (\operatorname{avg})$ & time & $\min (\operatorname{avg})$ & time \\
\hline sc-ldoor & $856936(856949)$ & 998 & $856755(856757.4)$ & 221.24 \\
\hline sc-msdoor & $381565(381567.1)$ & 958.01 & 381558(381558.9) & 18.38 \\
\hline sc-nasasrb & $51244(51246.9)$ & 768.2 & $51240(51243.2)$ & 475.53 \\
\hline sc-pkustk11 & 83911(83911) & 591.98 & $83912(83913.2)$ & 12.03 \\
\hline sc-pkustk13 & $89220(89222.5)$ & 938.54 & $89218(89220.3)$ & 392.48 \\
\hline sc-pwtk & 207741(207746.1) & 154.63 & 207716(207719.9) & 208.31 \\
\hline sc-shipsec 1 & $117482(117518.4)$ & 991.04 & $117281(117297.5)$ & 742.95 \\
\hline sc-shipsec5 & $147292(147314.2)$ & 985.78 & $147067(147077.4)$ & 548.72 \\
\hline soc-BlogCatalog & 20752(20752.2) & 424.94 & 20752(20752) & 0.45 \\
\hline soc-brightkite & 21191(21192.9) & 859.39 & $21190(21190.1)$ & 0.24 \\
\hline soc-buzznet & 30613(30613.7) & 580.13 & $30624(30624)$ & 6.27 \\
\hline soc-delicious & $85566(85573.2)$ & 659.65 & 85532(85536.1) & 1.69 \\
\hline soc-digg & $103312(103321.7)$ & 566.02 & $103242(103242)$ & 2.81 \\
\hline soc-dolphins & $34(34)$ & $<0.01$ & $34(34)$ & $<0.01$ \\
\hline soc-douban & $8685(8685)$ & 0.04 & $8685(8685)$ & 0.04 \\
\hline soc-epinions & 9757(9757) & 113.99 & 9757(9757) & 0.17 \\
\hline soc-flickr & $153343(153349.3)$ & 38.58 & 153271(153271.1) & 18.33 \\
\hline soc-flixster & 96318(96319.2) & 3.14 & $96317(96317)$ & 2.72 \\
\hline soc-FourSquare & 90131(90136.6) & 882.48 & $90109(90109.7)$ & 78.47 \\
\hline soc-gowalla & 84312(84321.4) & 961.51 & $84223(84223)$ & 26.96 \\
\hline soc-karate & $14(14)$ & $<0.01$ & $14(14)$ & $<0.01$ \\
\hline soc-lastfm & 78692(78695.1) & 591.63 & 78688(78688) & 1.14 \\
\hline soc-livejournal & 1882773(1882841.4) & 1000 & $1869002(1869007.6)$ & 934.25 \\
\hline soc-LiveMocha & $43430(43432.6)$ & 786.35 & 43427(43427) & 29.86 \\
\hline soc-orkut & 2193141(2193277.4) & 1000 & $2171246(2171290.2)$ & 997.16 \\
\hline soc-pokec & 844306(844329.2) & 610.7 & 843387(843390.4) & 641.83 \\
\hline soc-slashdot & 22373(22375.3) & 850.36 & 22373(22373) & 0.29 \\
\hline soc-twitter-follows & $2323(2323)$ & 0.22 & $2323(2323)$ & 0.17 \\
\hline soc-wiki-Vote & 406(406) & $<0.01$ & $406(406)$ & $<0.01$ \\
\hline soc-youtube & 146454(146461.6) & 47.1 & 146377(146377) & 5.27 \\
\hline soc-youtube-snap & 277012(277017.4) & 209.33 & 276947(276947) & 14.49 \\
\hline tech-as-caida2007 & $3683(3683)$ & 0.02 & $3683(3683)$ & $<0.01$ \\
\hline tech-as-skitter & $525950(525962.1)$ & 570.23 & 525538(525542.7) & 599.46 \\
\hline tech-internet-as & $5700(5700)$ & 19.47 & $5700(5700)$ & 0.04 \\
\hline tech-p2p-gnutella & 15682(15682) & 30.64 & 15682(15682) & $\mathbf{0 . 0 3}$ \\
\hline tech-RL-caida & 74763(74772.7) & 936.24 & $74710(74713.1)$ & 9.28 \\
\hline tech-routers-rf & $795(795)$ & $<0.01$ & $795(795)$ & $<0.01$ \\
\hline tech-WHOIS & $2284(2284)$ & 0.09 & 2284(2284) & $<\mathbf{0 . 0 1}$ \\
\hline web-arabic-2005 & 114454(114458.6) & 65.27 & $114426(114427.3)$ & 254.95 \\
\hline web-BerkStan & $5384(5384)$ & 9.53 & $5384(5384)$ & 23.13 \\
\hline web-edu & $1451(1451)$ & $<0.01$ & $1451(1451)$ & $<0.01$ \\
\hline web-google & 498(498) & $<0.01$ & $498(498)$ & $<0.01$ \\
\hline web-indochina-2004 & $7300(7300)$ & 14.03 & $7300(7300)$ & 0.12 \\
\hline web-it-2004 & 414705(414720.7) & 643.76 & 414688(414689.9) & 315 \\
\hline web-polblogs & $244(244)$ & $<0.01$ & 244(244) & $<0.01$ \\
\hline web-sk-2005 & $58206(58209.8)$ & 655.21 & 58173(58173) & 9.71 \\
\hline web-spam & 2297(2297) & 0.53 & 2297(2297) & 0.01 \\
\hline web-uk-2005 & 127774(127774) & 1.21 & 127774(127774) & 1.05 \\
\hline web-webbase-2001 & $2652(2652)$ & 1.39 & $2652(2652)$ & 0.02 \\
\hline web-wikipedia2009 & 649261(649310.7) & 999.16 & $648318(648325.1)$ & 667.81 \\
\hline
\end{tabular}

Langston, Suters, \& Symons, 2004). The fourth rule called Dominance Rule was introduced recently (Fomin, Grandoni, \& Kratsch, 2009). When using the reduction rules to simplify a graph, some vertices are fixed as covering vertices, if there must be an optimal vertex cover containing them. We use $G$ to denote the graph we are dealing with. 
- Degree-0 Rule: An isolated vertex $u$ (vertex of degree 0) cannot be in a vertex cover of optimal size. Thus, the graph $G$ can be simplified by deleting $u$.

- Degree-1 Rule: If a vertex $u$ is a pendant vertex (vertex of degree 1), there is a vertex cover of optimal size that does not contain the pendant vertex but does contain its unique neighbor $v$. Thus, vertex $v$ is fixed as a covering vertex, and $G$ is simplified by deleting both $u$ and $v$ and their incident edges.

- Degree-2 Rule: If there is a degree-two vertex $u$ with adjacent neighbors $v$ and $z$, then there is a vertex cover of optimal size that does not contain $u$ but does include both of these neighbors. Thus, vertex $v$ and $z$ are fixed as covering vertices, and $G$ is simplified by deleting $u, v, z$ and their incident edges.

- Dominance Rule: For two vertices $u$ and $v$, if $N[u] \subseteq N[v]$, we say vertex $v$ dominates vertex $u$. If a vertex $v$ dominates some vertex, there always exists a vertex cover of optimal size that contains $v$. Therefore, vertex $v$ is fixed as a covering vertex, and $G$ is simplified by deleting $v$ and its incident edges.

Note that Degree-1 and Degree-2 rules can be implied by the Dominance rule. However, these two rules can be executed much faster than the Dominance rule. Based on this observation, we develop a two-phase preprocessing algorithm for MinVC, which is called D1+D2+Dom. To implement the preprocessing algorithm, we use a queue, denoted as $Q$, to store the vertices to be processed by reduction rules, and a set $F$ to store the vertices fixed as covering vertices. The preprocessing algorithm consists of two phases, and in each phase it works in an iterative way. In the first phase, it simplifies the graph only using Degree-1 and Degree-2 rules (and Degree-0 rule). When the graph cannot be simplified anymore by only using Degree-1 and Degree-2 rules, the preprocessing algorithm enters the second phase, where the Dominance rule is used to simplify the graph iteratively until no more vertex can be deleted according to the rule.

The preprocessing algorithm returns a simplified graph and a set $F$ which includes all fixed covering vertices. Then, the simplified graph is taken as the input graph for a MinVC algorithm. Suppose the MinVC algorithm returns a vertex cover $C^{\prime}$ for the simplified graph, then $C^{*}=C^{\prime} \cup F$ is a vertex cover for the original graph and is returned as the solution found. Additionally, if after preprocessing, a graph becomes empty (i.e., $V(G)=\emptyset$ ), then the set $F$ is indeed a minimum vertex cover for the original graph.

\subsection{Effectiveness of Reduction Rules}

To examine the effects of the reduction rules, we implement and test three preprocessing algorithms, including D1, D1+D2, and D1+D2+Dom, which use different sets of reduction rules. As their names indicate, D1 only uses the Degree-1 rule, and D1+D2 uses both Degree-1 and Degree-2 rules, while D1+D2+Dom employs all the rules. Note that the trivial Degree-0 rule is executed in all the preprocessing algorithms.

The experiment results are listed in Tables 8 and 9. We can observe the significant effects of these reduction rules on the size of the graphs. Comparing the graph size after reduction, we can see that D1+D2+Dom produces smaller graphs than D1+D2, which in turn is better than D1. Regarding the run time, D1 and D1+D2 usually terminate within one second. For D1+D2+Dom, the run time is much longer for some instances. There are 3 instances for which D1+D2+Dom terminates in 
Table 8: Effectiveness study of reduction rules.

\begin{tabular}{|c|c|c|c|c|c|c|c|c|c|c|c|}
\hline \multirow{2}{*}{ instance } & \multirow{2}{*}{ IVI } & \multirow{2}{*}{$|\mathrm{E}|$} & \multicolumn{3}{|c|}{ D1 } & \multicolumn{3}{|c|}{$\mathrm{D} 1+\mathrm{D} 2$} & \multicolumn{3}{|c|}{ D1+D2+Dom } \\
\hline & & & $\left|V^{\prime}\right|$ & $\left|E^{\prime}\right|$ & time & $\left|V^{\prime}\right|$ & |E’| & time & |V'| & $\left|E^{\prime}\right|$ & time \\
\hline bio-celegans & 453 & 2025 & 441 & 1940 & $<0.01$ & 32 & 64 & $<0.01$ & 0 & 0 & $<0.01$ \\
\hline bio-diseasome & 516 & 1188 & 242 & 483 & $<0.01$ & 96 & 201 & $<0.01$ & 0 & 0 & $<0.01$ \\
\hline bio-dmela & 7393 & 25569 & 791 & 1393 & $<0.01$ & 750 & 1295 & $<0.01$ & 748 & 1285 & $<0.01$ \\
\hline bio-yeast & 1458 & 1948 & 59 & 79 & $<0.01$ & 15 & 24 & $<0.01$ & 8 & 8 & $<0.01$ \\
\hline ca-AstroPh & 17903 & 196972 & 14669 & 158845 & 0.01 & 7651 & 66272 & 0.01 & 0 & 0 & 0.01 \\
\hline ca-citeseer & 227320 & 814134 & 124478 & 447088 & 0.05 & 57470 & 262641 & 0.07 & 16 & 16 & 0.08 \\
\hline ca-coauthors-dblp & 540486 & 15245729 & 521385 & 14978514 & 0.07 & 495944 & 14555260 & 0.15 & 24 & 24 & 2.79 \\
\hline ca-CondMat & 21363 & 91286 & 14338 & 57252 & $<0.01$ & 5842 & 21081 & 0.01 & 0 & 0 & $<0.01$ \\
\hline ca-CSphd & 1882 & 1740 & 4 & 4 & $<0.01$ & 4 & 4 & $<0.01$ & 4 & 4 & $<0.01$ \\
\hline ca-dblp-2010 & 226413 & 716460 & 109343 & 317705 & 0.06 & 44355 & 156299 & 0.06 & 8 & 8 & 0.06 \\
\hline ca-dblp-2012 & 317080 & 1049866 & 143132 & 377759 & 0.13 & 49573 & 147140 & 0.13 & 29 & 29 & 0.12 \\
\hline ca-Erdos992 & 6100 & 7515 & 0 & 0 & $<0.01$ & 0 & 0 & $<0.01$ & 0 & 0 & $<0.01$ \\
\hline ca-GrQc & 4158 & 13422 & 1783 & 6600 & $<0.01$ & 574 & 3494 & $<0.01$ & 0 & 0 & $<0.01$ \\
\hline ca-HepPh & 11204 & 117619 & 6740 & 67573 & 0.01 & 2589 & 30501 & 0.01 & 4 & 4 & $<0.01$ \\
\hline ca-hollywood-2009 & 1069126 & 56306653 & 1042751 & 52438424 & 2.45 & 965676 & 40863964 & 9.19 & 13 & 13 & 35.73 \\
\hline ca-MathSciNet & 332689 & 820644 & 55851 & 92336 & 0.12 & 11678 & 24654 & 0.11 & 59 & 61 & 0.13 \\
\hline ca-netscience & 379 & 914 & 242 & 518 & $<0.01$ & 86 & 195 & $<0.01$ & 0 & 0 & $<0.01$ \\
\hline ia-email-EU & 32430 & 54397 & 3 & 3 & 0.01 & 0 & 0 & $<0.01$ & 0 & 0 & $<0.01$ \\
\hline ia-email-univ & 1133 & 5451 & 620 & 2206 & $<0.01$ & 382 & 1073 & $<0.01$ & 178 & 374 & $<0.01$ \\
\hline ia-enron-large & 33696 & 180811 & 13214 & 36771 & 0.01 & 3355 & 7207 & 0.01 & 21 & 22 & $<0.01$ \\
\hline ia-enron-only & 143 & 623 & 119 & 529 & $<0.01$ & 94 & 371 & $<0.01$ & 50 & 112 & $<0.01$ \\
\hline ia-fb-messages & 1266 & 6451 & 227 & 391 & $<0.01$ & 64 & 81 & $<0.01$ & 61 & 74 & $<0.01$ \\
\hline ia-infect-dublin & 410 & 2765 & 374 & 2497 & $<0.01$ & 349 & 2340 & $<0.01$ & 186 & 927 & $<0.01$ \\
\hline ia-infect-hyper & 113 & 2196 & 111 & 2098 & $<0.01$ & 111 & 2098 & $<0.01$ & 108 & 1883 & $<0.01$ \\
\hline ia-reality & 6809 & 7680 & 0 & 0 & $<0.01$ & 0 & 0 & $<0.01$ & 0 & 0 & $<0.01$ \\
\hline ia-wiki-Talk & 92117 & 360767 & 101 & 106 & 0.03 & 12 & 12 & 0.03 & 12 & 12 & 0.03 \\
\hline inf-power & 4941 & 6594 & 458 & 614 & $<0.01$ & 175 & 214 & $<0.01$ & 155 & 179 & $<0.01$ \\
\hline inf-roadNet-CA & 1957027 & 2760388 & 1062587 & 1571668 & 0.32 & 913469 & 1350136 & 0.34 & 907378 & 1335179 & 0.44 \\
\hline inf-roadNet-PA & 1087562 & 1541514 & 561133 & 839083 & 0.17 & 470492 & 704445 & 0.2 & 467671 & 697305 & 0.26 \\
\hline inf-road-usa & 23947347 & 28854312 & 5310709 & 7258703 & 6.13 & 4462295 & 6173990 & 6.72 & 4454350 & 6156614 & 8.02 \\
\hline rec-amazon & 91813 & 125704 & 33657 & 49524 & 0.02 & 6886 & 11038 & 0.01 & 1650 & 1789 & 0.01 \\
\hline rt-retweet & 96 & 117 & 0 & 0 & $<0.01$ & 0 & 0 & $<0.01$ & 0 & 0 & $<0.01$ \\
\hline rt-retweet-crawl & 1112702 & 2278852 & 517 & 1248 & 0.43 & 472 & 1178 & 0.43 & 415 & 693 & 0.43 \\
\hline rt-twitter-copen & 761 & 1029 & 14 & 15 & $<0.01$ & 0 & 0 & $<0.01$ & 0 & 0 & $<0.01$ \\
\hline socfb-A-anon & 3097165 & 23667394 & 1032 & 1660 & 5.45 & 276 & 659 & 4.34 & 127 & 174 & 4.51 \\
\hline socfb-B-anon & 2937612 & 20959854 & 479 & 733 & 4.28 & 130 & 204 & 3.94 & 81 & 91 & 3.77 \\
\hline socfb-Berkeley13 & 22900 & 852419 & 21474 & 778596 & 0.04 & 20771 & 735629 & 0.05 & 19561 & 645318 & 0.18 \\
\hline socfb-CMU & 6621 & 249959 & 6182 & 229918 & 0.01 & 5941 & 217267 & 0.01 & 5386 & 173161 & 0.04 \\
\hline socfb-Duke14 & 9885 & 506437 & 9328 & 475087 & 0.02 & 9010 & 452633 & 0.02 & 8403 & 399227 & 0.1 \\
\hline socfb-Indiana & 29732 & 1305757 & 28574 & 1252996 & 0.04 & 27928 & 1214330 & 0.06 & 26675 & 1096518 & 0.27 \\
\hline socfb-MIT & 6402 & 251230 & 5808 & 225360 & 0.01 & 5513 & 207675 & 0.01 & 4860 & 155166 & 0.04 \\
\hline socfb-OR & 63392 & 816886 & 42756 & 532334 & 0.05 & 34187 & 401175 & 0.07 & 25782 & 237632 & 0.16 \\
\hline socfb-Penn94 & 41536 & 1362220 & 39255 & 1267464 & 0.06 & 37992 & 1192146 & 0.08 & 35684 & 1042042 & 0.3 \\
\hline socfb-Stanford3 & 11586 & 568309 & 10421 & 506275 & 0.03 & 9846 & 473219 & 0.05 & 8995 & 399622 & 0.13 \\
\hline socfb-Texas84 & 36364 & 1590651 & 34932 & 1494828 & 0.06 & 34291 & 1449994 & 0.07 & 32661 & 1286733 & 0.33 \\
\hline socfb-uci-uni & 58790782 & 92208195 & 147 & 273 & 18.67 & 118 & 224 & 18.98 & 67 & 77 & 19.32 \\
\hline socfb-UCLA & 20453 & 747604 & 19062 & 689871 & 0.03 & 18404 & 656663 & 0.04 & 17251 & 567198 & 0.13 \\
\hline socfb-UConn & 17206 & 604867 & 16444 & 571019 & 0.01 & 16080 & 551369 & 0.02 & 15270 & 491637 & 0.11 \\
\hline socfb-UCSB37 & 14917 & 482215 & 14127 & 452899 & 0.02 & 13748 & 436144 & 0.02 & 13020 & 389722 & 0.08 \\
\hline socfb-UF & 35111 & 1465654 & 33847 & 1387729 & 0.05 & 33169 & 1341208 & 0.07 & 31385 & 1205668 & 0.32 \\
\hline socfb-UIllinois & 30795 & 1264421 & 29530 & 1204844 & 0.04 & 28866 & 1163750 & 0.06 & 27428 & 1056152 & 0.26 \\
\hline socfb-Wisconsin 87 & 23831 & 835946 & 22764 & 794760 & 0.03 & 22212 & 762643 & 0.04 & 20966 & 675559 & 0.19 \\
\hline
\end{tabular}

more than 10 seconds, and the longest run time reaches 35 seconds. Nevertheless, this is acceptable 
Table 9: Effectiveness study of reduction rules (continued).

\begin{tabular}{|c|c|c|c|c|c|c|c|c|c|c|c|}
\hline \multirow{2}{*}{ instance } & \multirow{2}{*}{$|\mathrm{V}|$} & \multirow{2}{*}{$|\mathrm{E}|$} & \multicolumn{3}{|c|}{ D1 } & \multicolumn{3}{|c|}{$\mathrm{D} 1+\mathrm{D} 2$} & \multicolumn{3}{|c|}{$\mathrm{D} 1+\mathrm{D} 2+\mathrm{Dom}$} \\
\hline & & & IV' & $\left|E^{\prime}\right|$ & time & IV'I & $\left|E^{\prime}\right|$ & time & $\left|V^{\prime}\right|$ & $\left|E^{\prime}\right|$ & time \\
\hline sc-ldoor & 952203 & 20770807 & 909537 & 20770807 & 0.1 & 909537 & 20770807 & 0.09 & 20301 & 34702 & 2.02 \\
\hline sc-msdoor & 415863 & 9378650 & 404785 & 9378650 & 0.03 & 404785 & 9378650 & 0.03 & 43041 & 120519 & 0.88 \\
\hline sc-nasasrb & 54870 & 1311227 & 54870 & 1311227 & $<0.01$ & 54870 & 1311227 & $<0.01$ & 15425 & 82814 & 0.12 \\
\hline sc-pkustk11 & 87804 & 2565054 & 87804 & 2565054 & $<0.01$ & 87804 & 2565054 & $<0.01$ & 3814 & 4362 & 0.24 \\
\hline sc-pkustk13 & 94893 & 3260967 & 94893 & 3260967 & $<0.01$ & 94893 & 3260967 & $<0.01$ & 14711 & 47933 & 0.36 \\
\hline sc-pwtk & 217891 & 5653221 & 217847 & 5653055 & $<0.01$ & 217847 & 5653055 & $<0.01$ & 38430 & 164598 & 0.48 \\
\hline sc-shipsec1 & 140385 & 1707759 & 140385 & 1707759 & $<0.01$ & 140376 & 1707739 & $<0.01$ & 111692 & 1060673 & 0.14 \\
\hline sc-shipsec5 & 179104 & 2200076 & 179092 & 2200067 & $<0.01$ & 179089 & 2200064 & $<0.01$ & 134066 & 1252861 & 0.18 \\
\hline soc-BlogCatalog & 88784 & 2093195 & 483 & 807 & 0.39 & 153 & 362 & 0.39 & 47 & 62 & 0.37 \\
\hline soc-brightkite & 56739 & 212945 & 3020 & 6654 & 0.02 & 511 & 2497 & 0.01 & 120 & 159 & 0.02 \\
\hline soc-buzznet & 101163 & 2763066 & 11375 & 37250 & 0.44 & 3169 & 8655 & 0.42 & 1881 & 4465 & 0.42 \\
\hline soc-delicious & 536108 & 1365961 & 18690 & 69612 & 0.16 & 18499 & 69131 & 0.14 & 18367 & 68559 & 0.19 \\
\hline soc-digg & 770799 & 5907132 & 2763 & 6951 & 1.5 & 848 & 3754 & 1.46 & 402 & 532 & 1.43 \\
\hline soc-dolphins & 62 & 159 & 40 & 82 & $<0.01$ & 24 & 41 & $<0.01$ & 18 & 21 & $<0.01$ \\
\hline soc-douban & 154908 & 327162 & 17 & 31 & 0.03 & 17 & 31 & 0.02 & 16 & 28 & 0.03 \\
\hline soc-er & 26588 & 100120 & 1580 & 3334 & 0.01 & 221 & 538 & $<0.01$ & 60 & 68 & $<0.01$ \\
\hline soc-flickr & 513969 & 3190452 & 31972 & 342883 & 0.67 & 8946 & 54497 & 56 & 3921 & 18367 & 0.57 \\
\hline soc-fll & 2523386 & 7918801 & 37 & 196 & 1.03 & 23 & 35 & & 8 & 8 & 1.21 \\
\hline soc-F & 39014 & 3214986 & 1836 & 2644 & 1.97 & 387 & 680 & & 138 & 149 & 34 \\
\hline soc- -8 & 196591 & 0327 & 31780 & 78356 & 0.11 & 4563 & 15489 & & 1176 & 4887 & 0.11 \\
\hline soc- -1 & 34 & 78 & 9 & 10 & $<0.01$ & 4 & 4 & $<0.01$ & 4 & 4 & $<0.01$ \\
\hline soc-lastfm & 1191805 & 4519330 & 167 & 232 & 0.71 & 32 & 45 & 0.6 & 17 & 18 & 0.71 \\
\hline soc-livej & 4033137 & 27933062 & 1220365 & 5202120 & 5.02 & 213667 & 1460786 & 4.87 & 41637 & 74057 & 5.07 \\
\hline soc-LiveMocha & 04103 & 2193083 & 655 & 732 & 0.34 & 24 & 24 & 0.36 & 24 & 24 & 0.35 \\
\hline soc-orkut & 2997166 & 106349209 & 2829189 & 95788825 & 3.83 & 2754327 & 91669427 & 5.38 & 2599169 & 82644648 & 34.28 \\
\hline soc-pokec & 1632803 & 22301964 & 1090351 & 12491982 & 2.46 & 963857 & 10265224 & 3.23 & 881359 & 8572011 & 8.06 \\
\hline soc-slashdot & 70068 & 58647 & 903 & 1167 & 0.03 & 112 & 214 & 0.04 & 26 & 27 & 0.04 \\
\hline soc-twitter-follows & 404719 & 713319 & 0 & 0 & 0.08 & 0 & 0 & & 0 & 0 & 0.08 \\
\hline soc-wiki-Vote & 889 & 2914 & 121 & 187 & $<0.01$ & 24 & 29 & $<0.01$ & 24 & 29 & $<0.01$ \\
\hline soc-you & 495957 & 1936748 & 8156 & 10894 & 0.31 & 1464 & 2553 & 0.28 & 535 & 610 & 0.28 \\
\hline soc-youtube-snap & 1134890 & 2987624 & 12035 & 15014 & 0.59 & 1584 & 2680 & 0.48 & 584 & 653 & 0.5 \\
\hline tech-as-caida2007 & 26475 & 53381 & 40 & 50 & $<0.01$ & 15 & 18 & $<0.01$ & 15 & 18 & $<0.01$ \\
\hline tech-as-skitter & 1694616 & 11094209 & 475124 & 1600224 & 1.56 & 262723 & 831476 & 1.28 & 228382 & 619077 & 1.36 \\
\hline tech-internet-as & 40164 & 85123 & 102 & 131 & $<0.01$ & 15 & 18 & $<0.01$ & 15 & 18 & $<0.01$ \\
\hline tech-p2p-gnutella & 62561 & 147878 & 38 & 54 & 0.01 & 38 & 54 & & 38 & 54 & 0.01 \\
\hline tech-1 & 190914 & 607610 & 70226 & 211590 & 0.06 & 51553 & 139142 & 07 & 47149 & 119062 & 0.1 \\
\hline & & & & 685 & $<0.01$ & 14 & 22 & & 4 & 4 & $<0.01$ \\
\hline tecl & 7476 & 56943 & 665 & 3409 & $<0.01$ & 294 & 1611 & $<0.01$ & 63 & 122 & $<0.01$ \\
\hline 2005 & 163598 & 1747269 & 102515 & 1560020 & 0.05 & 98504 & 1542669 & & 1994 & 3514 & 0.1 \\
\hline rkStan & 12305 & 500 & 5121 & 8345 & $<0.01$ & 1105 & 1589 & & 771 & 857 & $<0.01$ \\
\hline & & 6474 & 1 & 689 & $<0.01$ & 91 & 582 & & 0 & 0 & $<0.01$ \\
\hline & 1299 & 2773 & 326 & 1140 & $<0.01$ & 218 & 903 & $<0.01$ & 0 & 0 & $<0.01$ \\
\hline web-indochina-2004 & 11358 & 47606 & 6297 & 32421 & $<0.01$ & 5355 & 25973 & $<0.01$ & 12 & 12 & $<0.01$ \\
\hline web-it-2004 & 509338 & 7178413 & 424893 & 6440816 & 0.16 & 423389 & 6428764 & 0.16 & 4995 & 21441 & 1.17 \\
\hline web-polblogs & 643 & 2280 & 48 & 92 & $<0.01$ & 16 & 23 & $<0.01$ & 4 & 4 & $<0.01$ \\
\hline web-sk-2005 & 121422 & 334419 & 42237 & 225932 & 0.02 & 39152 & 218233 & 0.02 & 27775 & 158337 & 0.03 \\
\hline web-spam & 4767 & 37375 & 1971 & 8540 & 0.01 & 874 & 2397 & 0.01 & 214 & 312 & $<0.01$ \\
\hline web-uk-2005 & 129632 & 11744049 & 127716 & 11643622 & 0.04 & 127713 & 11643619 & 0.04 & 0 & 0 & 2.55 \\
\hline web-webbase-2001 & 16062 & 25593 & 2085 & 5203 & $<0.01$ & 1913 & 4870 & $<0.01$ & 1391 & 2458 & $<0.01$ \\
\hline web-wikipedia2009 & 1864433 & 4507315 & 154344 & 302990 & 0.74 & 37561 & 98257 & 0.68 & 18941 & 47389 & 0.96 \\
\hline
\end{tabular}

for such an NP-hard problem. Additionally, 15 graphs become empty after being simplified by D1+D2+Dom, which means D1+D2+Dom alone solves these instances exactly.

We also study how often a reduction rule is executed in the D1+D2+Dom preprocessing algorithm. For each instance class, we calculate the averaged percentage of each rule over all rules, 
Table 10: The percentage of executions for each reduction rule, which equals the number of executions of the rule divided by the number of executions of all rules.

\begin{tabular}{lrrr}
\hline instance class & D1 & D2 & Dom \\
\hline bio & $72.25 \%$ & $16.40 \%$ & $11.35 \%$ \\
ca & $41.02 \%$ & $18.89 \%$ & $40.09 \%$ \\
ia & $60.00 \%$ & $9.31 \%$ & $30.69 \%$ \\
inf & $94.25 \%$ & $5.15 \%$ & $0.60 \%$ \\
rec & $70.39 \%$ & $20.31 \%$ & $9.30 \%$ \\
rt & $97.95 \%$ & $2.04 \%$ & $0.01 \%$ \\
socfb & $43.16 \%$ & $10.41 \%$ & $46.43 \%$ \\
sc & $<0.01 \%$ & $<0.01 \%$ & $>99.99 \%$ \\
soc & $84.06 \%$ & $10.68 \%$ & $5.26 \%$ \\
tech & $89.27 \%$ & $7.99 \%$ & $2.74 \%$ \\
web & $51.65 \%$ & $8.83 \%$ & $39.52 \%$ \\
\hline
\end{tabular}

and the results are reported in Table 10. From these results, we have the following observations: For different instance classes (which can be regarded as graphs of different structures), the figures are considerably different. An extreme example is that the percentage of the Dominance rule is less than $1 \%$ for inf and $r t$ instance classes, while it is almost $100 \%$ for the sc class. The Degree- 1 rule usually occupies a large percentage over all rules, except one class. For 8 out of the total 11 classes, the Degree-1 rule's executions occupy more than a half of all executions of all rules. Overall, Degree-2 is the least often executed rule, compared to the Degree-1 rule and the Dominance rule. To some extent, we can consider it is less useful compared to the other two rules. For some instances such as the sc instances (one can also refer to the results on sc instances in Table 9), the Degree-1 and Degree-2 rules are almost useless, while the Dominance is more powerful and can simplify the graphs.

\subsection{Connected Components in Simplified Graphs}

In this subsection, we study the structure of the simplified graphs produced by the preprocessing algorithm. Specifically, we investigate the connected components in the simplified graph. The results are presented in Tables 11, where we report the number of connected components ('\#comp.') and the size of the largest connected component (' $\left|V^{*}\right|$ ' and ' $\left|E^{*}\right|$ '). We do not report the results for the small graphs with fewer than 2000 vertices, as well as the graphs reduced to become empty by D1+D2+Dom.

Seen from the results, many graphs are decomposed into small connected components after preprocessing. For 58\% of the graphs, the largest connected component has fewer than 2000 vertices, and thus can be solved by iteratively calling exact solvers on each component. Nevertheless, for the remaining $42 \%$ of the graphs, the largest connected component is still large and usually beyond the reach of exact solvers.

\subsection{Comparison with State of the Art Preprocessing Algorithm}

In recent years, reduction techniques for vertex cover and related problems have attracted more and more interest and have shown their power in practice (Akiba \& Iwata, 2016; Lamm, Sanders, Schulz, Strash, \& Werneck, 2016; Strash, 2016; Verma, Buchanan, \& Butenko, 2015; Cai \& Lin, 2016). Very recently, Strash developed a preprocessing algorithm named Simple for the maximum 
Table 11: Connected components in simplified graphs by D1+D2+Dom.

\begin{tabular}{lrrr}
\hline & & \multicolumn{2}{c}{ largest component } \\
\cline { 2 - 4 } instance & & $\left|V^{*}\right|$ & $\left|E^{*}\right|$ \\
\hline bio-dmela & 15 & 686 & 1217 \\
ca-citeseer & 4 & 4 & 4 \\
ca-coauthors-dblp & 6 & 4 & 4 \\
ca-dblp-2010 & 2 & 4 & 4 \\
ca-dblp-2012 & 7 & 5 & 5 \\
ca-HepPh & 1 & 4 & 4 \\
ca-hollywood-2009 & 3 & 5 & 5 \\
ca-MathSciNet & 14 & 6 & 7 \\
ia-enron-large & 4 & 8 & 9 \\
ia-wiki-Talk & 3 & 4 & 4 \\
inf-power & 18 & 19 & 28 \\
inf-roadNet-CA & 4047 & 384123 & 577173 \\
inf-roadNet-PA & 2129 & 234989 & 359992 \\
inf-road-usa & 89584 & 58524 & 93033 \\
rec-amazon & 316 & 25 & 32 \\
rt-retweet-crawl & 48 & 62 & 120 \\
socfb-A-anon & 26 & 15 & 45 \\
socfb-B-anon & 18 & 8 & 13 \\
socfb-Berkeley13 & 1 & 19561 & 645318 \\
socfb-CMU & 1 & 5386 & 173161 \\
socfb-Duke14 & 1 & 8403 & 399227 \\
socfb-Indiana & 1 & 26675 & 1096518 \\
socfb-MIT & 1 & 4860 & 155166 \\
socfb-OR & 2 & 25778 & 237628 \\
socfb-Penn94 & 1 & 35684 & 1042042 \\
socfb-Stanford3 & 2 & 8991 & 399618 \\
socfb-Texas84 & 1 & 32661 & 1286733 \\
socfb-uci-uni & 14 & 8 & 12 \\
socfb-UCLA & 1 & 17251 & 567198 \\
socfb-UConn & 1 & 15270 & 491637 \\
socfb-UCSB37 & 1 & 13020 & 389722 \\
socfb-UF & 1 & 31385 & 1205668 \\
socfb-UIllinois & 1 & 27428 & 1056152 \\
socfb-Wisconsin87 & 1 & 20966 & 675559 \\
sc-ldoor & 105 & 4902 & 7109 \\
sc-msdoor & 6 & 35057 & 100094 \\
sc-nasasrb & 8 & 14832 & 81654 \\
sc-pkustk11 & 196 & 388 \\
\hline & & & \\
\hline
\end{tabular}

\begin{tabular}{lrrr}
\hline instance & & \multicolumn{2}{c}{ largest component } \\
\cline { 3 - 4 } & & $\left|V^{*}\right|$ & $\left|E^{*}\right|$ \\
\hline sc-pkustk13 & 1 & 14711 & 47933 \\
sc-pwtk & 76 & 36336 & 162504 \\
sc-shipsec1 & 3 & 111515 & 1059903 \\
sc-shipsec5 & 30 & 133624 & 1251679 \\
soc-BlogCatalog & 7 & 15 & 22 \\
soc-brightkite & 16 & 22 & 51 \\
soc-buzznet & 6 & 1849 & 4386 \\
soc-delicious & 754 & 1914 & 8039 \\
soc-digg & 71 & 29 & 44 \\
soc-douban & 1 & 16 & 28 \\
soc-epinions & 11 & 17 & 25 \\
soc-flickr & 147 & 3043 & 17179 \\
soc-flixster & 2 & 4 & 4 \\
soc-FourSquare & 30 & 8 & 9 \\
soc-gowalla & 79 & 777 & 4449 \\
soc-lastfm & 4 & 5 & 6 \\
soc-livejournal & 3536 & 16517 & 28617 \\
soc-LiveMocha & 6 & 4 & 4 \\
soc-orkut & 5 & 2599153 & 82644632 \\
soc-pokec & 10 & 881319 & 8571967 \\
soc-slashdot & 6 & 6 & 7 \\
soc-youtube & 123 & 22 & 82 \\
soc-youtube-snap & 131 & 17 & 50 \\
tech-as-caida2007 & 2 & 9 & 12 \\
tech-as-skitter & 11158 & 108182 & 367812 \\
tech-internet-as & 3 & 7 & 10 \\
tech-p2p-gnutella & 5 & 12 & 20 \\
tech-RL-caida & 1663 & 34528 & 98587 \\
tech-routers-rf & 1 & 4 & 4 \\
tech-WHOIS & 6 & 26 & 61 \\
web-arabic-2005 & 84 & 483 & 855 \\
web-BerkStan & 83 & 306 & 365 \\
web-indochina-2004 & 3 & 4 & 4 \\
web-it-2004 & 128 & 156 & 635 \\
web-sk-2005 & 469 & 877 & 4569 \\
web-spam & 4 & 199 & 295 \\
web-webbase-2001 & 5 & 1365 & 2422 \\
web-wikipedia2009 & 1130 & 2289 & 5871 \\
\hline & & &
\end{tabular}

independent set (MaxIS) problem, which consists only of a small suite of simple reductions, which, however, are very effective (Strash, 2016). In this subsection, we compare our preprocessing algorithm D1+D2+Dom for MinVC with Strash's preprocessing algorithm dubbed Simple for MaxIS, by comparing the size of the simplified graph. Bear in mind that the simplified graphs produced by Simple should be solved by a MaxIS algorithm, since its reduction rules are designed for MaxIS.

The comparison results are summarized in Table 12. The instances that are reduced to empty by both algorithms are not listed in the table. Overall, the two preprocessing algorithms have similar performance and are complementary to each other on these real-world instances. Specifically, our preprocessing algorithm is more effective on Facebook networks and scientific computation networks, while the MaxIS preprocessing algorithm Simple is more effective on infrastructure 
Table 12: Comparing D1+D2+Dom with the MaxIS preprocessing algorithm Simple.

\begin{tabular}{|c|c|c|c|c|c|c|c|c|c|c|c|c|c|}
\hline \multirow{2}{*}{ instance } & \multicolumn{3}{|c|}{ Simple } & \multicolumn{3}{|c|}{ D1+D2+Dom } & \multirow{2}{*}{ instance } & \multicolumn{3}{|c|}{ Simple } & \multicolumn{3}{|c|}{ D1+D2+Dom } \\
\hline & $\left|V^{\prime}\right|$ & $\left|E^{\prime}\right|$ & time & $\left|V^{\prime}\right|$ & $\left|E^{\prime}\right|$ & time & & $\left|V^{\prime}\right|$ & $\left|E^{\prime}\right|$ & time & | I' & $\left|\mathrm{E}^{\prime}\right|$ & time \\
\hline o-dmela & 7 & 12 & 0.01 & 748 & 1285 & $<0.01$ & sc-pkustk11 & 55452 & 1541478 & 0.07 & 3814 & 4362 & 0.24 \\
\hline bio-yeast & $\mathbf{0}$ & & $<0.01$ & 8 & & $<0.01$ & sc-pkustk13 & 69918 & 2228836 & 0.06 & 14711 & 47933 & 0.36 \\
\hline ca-citeseer & 5 & 8 & 0.12 & 16 & 16 & 0.08 & sc-pwtk & 217416 & 5647897 & 0.05 & 38430 & 164598 & 0.48 \\
\hline ca-authors-dblp & 27 & 67 & 1.25 & 24 & 24 & 2.79 & sc-shipsec1 & 139660 & 1702022 & 0.01 & 111692 & 1060673 & 0.14 \\
\hline ca-CSphd & $\mathbf{0}$ & & $<0.01$ & 4 & & $<0.01$ & sc-shipsec5 & 175205 & 2155554 & 0.02 & 134066 & 1252861 & 0.18 \\
\hline ca-dblp-2010 & $\mathbf{0}$ & $\mathbf{0}$ & 0.09 & 8 & 8 & 0.06 & soc-BlogCatalog & 6 & 12 & 0.71 & 47 & 62 & 0.37 \\
\hline ca-dblp-2012 & 13 & 24 & 0.21 & 29 & 29 & 0.12 & soc-brightkite & 24 & 66 & 0.04 & 120 & 159 & 0.02 \\
\hline ca-HepPh & 7 & 17 & 0.01 & 4 & & $<0.01$ & soc-buzznet & 237 & 1920 & 5.39 & 1881 & 4465 & 0.42 \\
\hline ca-hollywood-09 & 9 & 28 & 15.37 & 13 & 13 & 35.73 & soc-delicious & 11447 & 51092 & 0.26 & 18367 & 68559 & 0.19 \\
\hline ca-MathSciNet & $\mathbf{0}$ & $\mathbf{0}$ & 0.21 & 59 & 61 & 0.13 & soc-digg & 163 & 2272 & 2.68 & 402 & 532 & 1.43 \\
\hline ia-email-univ & 101 & 296 & $<0.01$ & 178 & 374 & $<0.01$ & soc-dolphins & $\mathbf{0}$ & & $<0.01$ & 18 & 21 & $<0.01$ \\
\hline ia-enron-large & 6 & & 0.02 & 21 & & $<0.01$ & soc-do & $\mathbf{0}$ & $\mathbf{0}$ & 0.07 & 16 & 28 & 0.03 \\
\hline ia-enron-only & 78 & 285 & $<0.01$ & 50 & 112 & $<0.01$ & soc- & 41 & 131 & 0.01 & 60 & 68 & $<0.01$ \\
\hline ia-fb-messages & $\mathbf{0}$ & & $<0.01$ & 61 & & $<0.01$ & soc & 1958 & 11948 & 1.46 & 3921 & 18367 & 0.57 \\
\hline ia-infect- & 263 & 1878 & $<0.01$ & 186 & 927 & $<0.01$ & soc & $\mathbf{0}$ & $\mathbf{0}$ & 1.57 & 8 & 8 & 1.21 \\
\hline ia-infect-hyper & 111 & 2098 & $<0.01$ & 108 & 1883 & $<0.01$ & soc & 29 & 81 & 8.3 & 138 & 149 & 1.34 \\
\hline ia-wiki-Talk & $\mathbf{0}$ & 0 & 0.06 & 12 & 12 & 0.03 & soc-gow: & 765 & 5556 & 0.62 & 1176 & 4887 & 0.11 \\
\hline inf-power & $\mathbf{0}$ & & $<0.01$ & 155 & 179 & $<0.01$ & soc-karate & $\mathbf{0}$ & & $<0.01$ & 4 & & $<0.01$ \\
\hline inf-roadNet-CA & 305166 & $\mathbf{5 3 9 7 3 5}$ & 0.58 & 907378 & 1335179 & 0.44 & soc-lastfm & $\mathbf{0}$ & $\mathbf{0}$ & 0.82 & 17 & 18 & 0.71 \\
\hline inf-roadNet-PA & 169050 & 298800 & 0.32 & 467671 & 697305 & 0.26 & soc-live & 28694 & 471112 & 11.97 & 41637 & 74057 & 5.07 \\
\hline inf-road-usa & 907352 & 1610582 & 11.32 & 4454350 & 6156614 & 8.02 & soc-LiveMocha & 0 & o & 3.26 & 24 & 24 & 0.35 \\
\hline rec-amazon & 489 & 869 & 0.03 & 1650 & 1789 & 0.01 & soc-orkut & 2623684 & 87854637 & 19.89 & 2599169 & 82644648 & 34.28 \\
\hline rt-retweet-crawl & 60 & 123 & 0.99 & 415 & 693 & 0.43 & soc-pokec & 748755 & 9207514 & 11.72 & 881359 & 8572011 & 8.06 \\
\hline socfb- & 27 & 109 & 7.35 & 127 & 174 & 4.51 & soc-sl & 0 & O & 0.1 & 26 & 27 & 0.04 \\
\hline socfb-B-anon & 5 & 8 & 5.74 & 81 & 91 & 3.77 & soc-wiki-Vote & $\mathbf{0}$ & & $<0.01$ & 24 & 29 & $<0.01$ \\
\hline socfb-Berkeley 13 & 319706 & 699207 & 0.07 & 19561 & 645318 & 0.18 & soc-youtube & 159 & 366 & 0.89 & 535 & 610 & 0.28 \\
\hline socfb-CMU & 5544 & 198448 & 0.01 & 5386 & 73161 & 0.04 & soc-youtube-snap & 76 & 212 & 1.68 & 584 & 653 & 0.5 \\
\hline socfb-Duke14 & 8525 & 429456 & 0.02 & 8403 & 399227 & 0.1 & tech-as- & $\mathbf{0}$ & $\mathbf{0}$ & 0.02 & 15 & 18 & $<0.01$ \\
\hline socfb-Indiana & 26926 & 1169133 & 0.06 & 26675 & 1096518 & 0.27 & tech-as-skitter & 65334 & 270110 & 9.36 & 228382 & 619077 & 1.36 \\
\hline socfb-MIT & 5103 & 186191 & 0.01 & 4860 & 155166 & 0.04 & & $\mathbf{0}$ & 0 & 0.02 & 15 & 18 & $<0.01$ \\
\hline socf & 24572 & 775 & 0.17 & 25782 & 237632 & 0.16 & & $\mathbf{0}$ & $\mathbf{0}$ & 0.03 & 38 & 54 & 0.01 \\
\hline enn94 & 36200 & 1136060 & 0.09 & 35684 & 1042042 & 0.3 & tech-RL-caida & 9248 & 27211 & 0.23 & 47149 & 119062 & 0.1 \\
\hline $\operatorname{tanford} 3$ & 9126 & 445324 & 0.04 & 8995 & 399622 & 0.13 & tech-ro & 0 & & $<0.01$ & 4 & & $<0.01$ \\
\hline as 84 & 32995 & 1372652 & 0.09 & 32661 & 1286733 & 0.33 & tech- $\mathrm{W}$ & 91 & 624 & 0.01 & 63 & 122 & $<0.01$ \\
\hline socfb-uci-uni & o & 0 & 32.83 & 67 & 77 & 19.32 & web-arabic-2005 & 328 & 1216 & 0.14 & 1994 & 3514 & 0.1 \\
\hline socfb-UCLA & 17327 & 616456 & 0.04 & 17251 & 567198 & 0.13 & web-BerkStan & $\mathbf{0}$ & & $<0.01$ & 771 & $857<$ & $<0.01$ \\
\hline socfb-UConn & 15435 & 527538 & 0.03 & 15270 & 491637 & 0.11 & web-indochina-04 & 36 & 171 & $<0.01$ & 12 & & $<0.01$ \\
\hline socfb-UCSB37 & 13074 & 413812 & 0.02 & 13020 & 389722 & 0.08 & web-it-2004 & 3381 & 20368 & 0.38 & 4995 & 21441 & 1.17 \\
\hline socfb-UF & 31872 & 1285610 & 0.09 & 31385 & 1205668 & 0.32 & web-polblogs & 0 & & $<0.01$ & 4 & & $<0.01$ \\
\hline socfb-UIllinois & 27786 & 1121816 & 0.05 & 27428 & 1056152 & 0.26 & web-sk-2005 & 24927 & 155059 & 0.04 & 27775 & 158337 & 0.03 \\
\hline socfb-Wiscsin87 & 21138 & 720010 & 0.05 & 20966 & 675559 & 0.19 & web-spam & 95 & 281 & 0.01 & 214 & $312<$ & $<0.01$ \\
\hline sc-ldoor & 9095372 & 20770807 & 0.14 & 20301 & 34702 & 2.02 & web-webbase-01 & 572 & 1237 & 0.01 & 1391 & $2458<$ & $<0.01$ \\
\hline sc-msdoor & 404785 & 9378650 & 0.06 & 43041 & 120519 & 0.88 & web-wikipedia09 & 9266 & 37579 & 2.53 & 18941 & 47389 & 0.96 \\
\hline sc-nasasrb & 54486 & 1305519 & 0.01 & 15425 & 82814 & 0.12 & & & & & & & \\
\hline
\end{tabular}

networks, technological networks, and web linkage networks. For the remaining instances, the two preprocessing algorithms have similar performance.

\subsection{The NuMVC2+p and FastVC2+p Solvers}

Preprocessing techniques can improve the performance of MinVC algorithms. We integrate the preprocessor D1+D2+Dom into the NuMVC2 and FastVC2 algorithms, resulting in two MinVC solvers called NuMVC2+p and FastVC2+p. These solvers call D1+D2+Dom to simplify the input 
Table 13: Experiment results of NuMVC2+p and FastVC2+p.

\begin{tabular}{|c|c|c|c|c|}
\hline \multirow{2}{*}{ instance } & \multicolumn{2}{|l|}{ NuMVC2+p } & \multicolumn{2}{|l|}{ FastVC2+p } \\
\hline & $\min (\operatorname{avg})$ & time & $\min (\mathrm{avg})$ & time \\
\hline bio-celegans & $249(249)$ & $<0.01$ & $249(249)$ & $<0.01$ \\
\hline bio-diseasome & $285(285)$ & $<0.01$ & $285(285)$ & $<0.01$ \\
\hline bio-dmela & $2630(2630)$ & $<\mathbf{0 . 0 1}$ & $2630(2630)$ & 1.34 \\
\hline bio-yeast & $456(456)$ & $<0.01$ & $456(456)$ & $<0.01$ \\
\hline ca-AstroPh & 11483(11483) & 0.01 & 11483(11483) & 0.01 \\
\hline ca-citeseer & 129193(129193) & 0.08 & 129193(129193) & 0.08 \\
\hline ca-coauthors-dblp & $472179(472179)$ & 2.77 & $472179(472179)$ & 2.79 \\
\hline ca-CondMat & $12480(12480)$ & $<0.01$ & $12480(12480)$ & $<0.01$ \\
\hline ca-CSphd & $550(550)$ & $<0.01$ & $550(550)$ & $<0.01$ \\
\hline ca-dblp-2010 & $121969(121969)$ & 0.06 & $121969(121969)$ & 0.06 \\
\hline ca-dblp-2012 & 164949(164949) & 0.13 & 164949(164949) & 0.12 \\
\hline ca-Erdos992 & $461(461)$ & $<0.01$ & $461(461)$ & $<0.01$ \\
\hline ca-GrQc & $2208(2208)$ & $<0.01$ & $2208(2208)$ & $<0.01$ \\
\hline ca-HepPh & $6555(6555)$ & $<0.01$ & $6555(6555)$ & $<0.01$ \\
\hline ca-hollywood-2009 & $864052(864052)$ & 35.58 & $864052(864052)$ & 35.73 \\
\hline ca-MathSciNet & 139951(139951) & 0.13 & 139951(139951) & 0.13 \\
\hline ca-netscience & $214(214)$ & $<0.01$ & $214(214)$ & $<0.01$ \\
\hline ia-email-EU & $820(820)$ & $<0.01$ & $820(820)$ & $<0.01$ \\
\hline ia-email-univ & $594(594)$ & $<0.01$ & $594(5$ & $<0.01$ \\
\hline ia-enron-large & $12781(12781)$ & $<0.01$ & $12781(12781)$ & $<0.01$ \\
\hline ia-enron-only & $86(86)$ & $<0.01$ & $86(86)$ & $<0.01$ \\
\hline ia-fb-messages & $578(578)$ & $<0.01$ & $578(578)$ & $<0.01$ \\
\hline ia-infect-dublin & $293(293)$ & $<0.01$ & 293(293) & $<0.01$ \\
\hline ia-infect-hyper & $90(90)$ & $<0.01$ & $90(90)$ & $<0.01$ \\
\hline ia-reality & $81(81)$ & $<0.01$ & $81(81)$ & $<0.01$ \\
\hline ia-wiki-Talk & $17288(17288)$ & 0.03 & $17288(17288)$ & 0.03 \\
\hline inf-power & 2203(2203) & $<0.01$ & $2203(2203)$ & $<0.01$ \\
\hline inf-roadNet-CA & $1005377(1005475.4)$ & 615.65 & $1001065(1001109.7)$ & 514.3 \\
\hline inf-roadNet-PA & $557312(557366.9)$ & 158.76 & $555046(555082.9)$ & 250.17 \\
\hline inf-road-usa & $11654069(11654194.8)$ & 1000 & $11527630(11527731.7)$ & 968.01 \\
\hline rec-amazon & $47605(47605)$ & 0.02 & $47605(47605)$ & $\mathbf{0 . 0 1}$ \\
\hline rt-retweet & $32(32)$ & $<0.01$ & $32(32)$ & $<0.01$ \\
\hline rt-retweet-crawl & $81040(81040)$ & 0.43 & $81040(81040)$ & 0.43 \\
\hline rt-twitter-copen & $237(237)$ & $<0.01$ & $237(237)$ & $<0.01$ \\
\hline socfb-A-anon & $375230(375230)$ & 4.50 & $375230(375230)$ & 4.51 \\
\hline socfb-B-anon & $303048(303048)$ & 3.75 & $303048(303048)$ & 3.77 \\
\hline socfb-Berkeley 13 & $17214(17217.3)$ & 742.83 & $17210(17211.9)$ & 369.86 \\
\hline socfb-CMU & 4986(4986) & 130.82 & 4986(4986.6) & 104.63 \\
\hline socfb-Duke14 & $7683(7683.3)$ & 241.79 & $7683(7683)$ & 201.17 \\
\hline socfb-Indiana & $23320(23327)$ & 645.3 & $23315(23317.1)$ & 524.84 \\
\hline socfb-MIT & $4657(4657)$ & 3.25 & $4657(4657)$ & 4.53 \\
\hline socfb-OR & $36551(36553.1)$ & 571.88 & $36548(36548.4)$ & 74.84 \\
\hline socfb-Penn94 & $31181(31183.7)$ & 606.92 & $31161(31164)$ & 414.21 \\
\hline socfb-Stanford3 & $8518(8518)$ & 6.35 & $8518(8518)$ & 1.69 \\
\hline socfb-Texas84 & $28177(28182.9)$ & 570.79 & $28166(28170.8)$ & 481.56 \\
\hline socfb-uci-uni & 866766(866766) & 18.57 & $866766(866766)$ & 19.32 \\
\hline socfb-UCLA & $15225(15226.4)$ & 466.85 & $15223(15224.1)$ & 242.24 \\
\hline socfb-UConn & 13233(13233.7) & 442.06 & 13230(13231.7) & 188.83 \\
\hline socfb-UCSB37 & $11261(11262.1)$ & 327.93 & $11261(11262.1)$ & 395.18 \\
\hline socfb-UF & $27320(27323.1)$ & 544.18 & 27306(27308.4) & 370.66 \\
\hline socfb-UIllinois & $24104(24105.9)$ & 529.50 & 24092(24093.6) & 350.65 \\
\hline socfb-Wisconsin 87 & 18389(18391.1) & 541.04 & $18383(18384.6)$ & 408.83 \\
\hline
\end{tabular}


Table 14: Experiment results of NuMVC2+p and FastVC2+p (continued).

\begin{tabular}{|c|c|c|c|c|}
\hline \multirow{2}{*}{ instance } & \multicolumn{2}{|l|}{ NuMVC2+p } & \multicolumn{2}{|l|}{ FastVC2+p } \\
\hline & $\min (\operatorname{avg})$ & time & $\min (\operatorname{avg})$ & time \\
\hline sc-ldoor & $856754(\mathbf{8 5 6 7 5 4})$ & 81.93 & $856754(856754.5)$ & 397.38 \\
\hline sc-msdoor & $381558(\mathbf{3 8 1 5 5 8})$ & 560.29 & $381558(381559.2)$ & 76.89 \\
\hline sc-nasasrb & $51248(51249.9)$ & 330.27 & $51239(51239.2)$ & 275.69 \\
\hline sc-pkustk11 & 83911(83911) & 0.56 & 83911(83911) & 1.92 \\
\hline sc-pkustk13 & $89221(89221.6)$ & 294.28 & $89227(89228.5)$ & 51.87 \\
\hline sc-pwtk & 207684(207695.9) & 449.29 & $207673(207681.9)$ & 796.31 \\
\hline sc-shipsec1 & 117282(117326.1) & 997.78 & $117276(117292.4)$ & 893.55 \\
\hline sc-shipsec5 & 147131(147152.1) & 995.17 & $147043(147055.2)$ & 545.29 \\
\hline soc-BlogCatalog & 20752(20752) & 0.36 & 20752(20752) & 0.37 \\
\hline soc-brightkite & $21190(21190)$ & 0.02 & $21190(21190)$ & 0.02 \\
\hline soc-buzznet & 30613(30613.6) & 0.46 & 30613(30613) & 0.44 \\
\hline soc-delicious & 85383(85391.6) & 550.74 & $85341(85342.7)$ & 537.59 \\
\hline soc-digg & 103234(103234) & 1.45 & $103234(103234)$ & 1.43 \\
\hline soc-dolphins & $34(34)$ & $<0.01$ & $34(34)$ & $<0.01$ \\
\hline soc-douban & $8685(8685)$ & 0.03 & $8685(8685)$ & 0.03 \\
\hline soc-epinions & 9757(9757) & $<0.01$ & 9757(9757) & $<0.01$ \\
\hline soc-flickr & 153271(153271) & 0.91 & 153271(153271) & 0.59 \\
\hline soc-flixster & 96317(96317) & 1.25 & 96317(96317) & 1.21 \\
\hline soc-FourSquare & $90108(90108)$ & 1.38 & $90108(90108)$ & 1.34 \\
\hline soc-gowalla & $84222(84222)$ & 0.12 & $84222(84222)$ & 1.17 \\
\hline soc-karate & $14(14)$ & $<0.01$ & $14(14)$ & $<0.01$ \\
\hline soc-lastfm & 78688(78688) & 0.73 & 78688(78688) & 0.71 \\
\hline soc-livejournal & $1868903(1868903.2)$ & 619.11 & 1868917(1868918.4) & 5.25 \\
\hline soc-LiveMocha & $43427(43427)$ & $\mathbf{0 . 3 3}$ & $43427(43427)$ & 0.35 \\
\hline soc-orkut & $2190339(2190777.1)$ & 999.8 & $2171200(2171236.4)$ & 996.03 \\
\hline soc-pokec & 844272(844306.2) & 204.47 & 843377(843380.4) & 574.95 \\
\hline soc-slashdot & $22373(22373)$ & 0.05 & $22373(22373)$ & 0.04 \\
\hline soc-twitter-follows & 2323(2323) & 0.08 & 2323(2323) & 0.08 \\
\hline soc-wiki-Vote & 406(406) & $<0.01$ & 406(406) & $<0.01$ \\
\hline soc-youtube & 146376(146376) & 0.28 & $146376(146376)$ & 0.28 \\
\hline soc-youtube-snap & $276945(276945)$ & 0.50 & $276945(276945)$ & 0.51 \\
\hline tech-as-caida2007 & $3683(3683)$ & $<0.01$ & $3683(3683)$ & $<0.01$ \\
\hline tech-as-skitter & $525163(525187.4)$ & 963.41 & $525269(525277.9)$ & 596.61 \\
\hline tech-internet-as & $5700(5700)$ & $<0.01$ & $5700(5700)$ & $<0.01$ \\
\hline tech-p2p-gnutella & $15682(15682)$ & $<\mathbf{0 . 0 1}$ & $15682(15682)$ & 0.01 \\
\hline tech-RL-caida & 74593(74597) & 832.06 & 74651(74654) & 862.06 \\
\hline tech-routers-rf & 795(795) & $<0.01$ & 795(795) & $<0.01$ \\
\hline tech-WHOIS & $2284(2284)$ & $<0.01$ & $2284(2284)$ & $<0.01$ \\
\hline web-arabic-2005 & $114420(114420)$ & 0.12 & 114420(114420) & 0.10 \\
\hline web-BerkStan & $5384(5384)$ & $<0.01$ & $5384(5384)$ & $<0.01$ \\
\hline web-edu & 1451(1451) & $<0.01$ & 1451(1451) & $<0.01$ \\
\hline web-google & 498(498) & $<0.01$ & 498(498) & $<0.01$ \\
\hline web-indochina-2004 & $7300(7300)$ & $<0.01$ & $7300(7300)$ & $<0.01$ \\
\hline web-it-2004 & $414507(414510.2)$ & 19.71 & $414528(414528)$ & 281.47 \\
\hline web-polblogs & $244(244)$ & $<0.01$ & $244(244)$ & $<0.01$ \\
\hline web-sk-2005 & $58173(58173.4)$ & 86.29 & $58173(\mathbf{5 8 1 7 3})$ & 1.41 \\
\hline web-spam & 2297(2297) & $<0.01$ & 2297(2297) & $<0.01$ \\
\hline web-uk-2005 & 127774(127774) & 2.55 & 127774(127774) & 2.55 \\
\hline web-webbase-2001 & $2651(2651)$ & 240.22 & $2652(2652)$ & $<0.01$ \\
\hline web-wikipedia2009 & $648294(648294)$ & 112.3 & 648305(648314.9) & 1.76 \\
\hline
\end{tabular}

graph to a smaller graph, which is then solved by the local search algorithm. Finally, the vertices that have been fixed in the preprocessing procedure and the vertex cover returned by the local search algorithm for the simplified graph compose a vertex cover for the original graph.

Experiment results of FastVC2+p and NuMVC2+p are reported in Tables 13 and 14. We firstly compare FastVC2 $2+p$ and NuMVC2 $+p$, with the aim of trying to establish the latest state of the art in heuristic solvers for MinVC on massive sparse graphs. The experiments are conducted on the whole 
Table 15: Comparison of NuMVC2+p against NuMVC2, and FastVC2+p against FastVC2.

\begin{tabular}{cccc}
\hline & NuMVC2+p vs NuMVC2 & & FastVC2+p vs FastVC2 \\
\cline { 2 - 2 } \#better-solution & 52 & & 37 \\
\#equal-solution & 44 & 60 \\
\#worse-solution & 3 & 5 \\
\hline
\end{tabular}

benchmark suite, and are based on the experiment protocol in Section 2.3. Note that the run time of NuMVC2 $+p$ and FastVC2 $+p$ include both the run time of D1+D2+Dom and that of the local search algorithm. The comparison results show that these two MinVC solvers are competitive with each other, and FastVC2+p has overall better performance. Specifically, with respect to solution quality, FastVC2 $2+p$ performs better than NuMVC $2+p$ on 23 instances, while NuMVC $2+p$ is better on 10 instances. For the remaining 69 instances, the two solvers obtain the same quality solutions. As for run time, the averaged run time over all runs on all 102 instances for FastVC2+p is 126 seconds, which is faster than that for NuMVC2+p (162 seconds).

We are also interested in the improvement due to the preprocessing algorithm on NuMVC2 and FastVC2. To this end, we compare the two solvers with preprocessing against their original algorithms. That is, we compare NuMVC2+p against NuMVC2, and compare FastVC2+p against FastVC2, with focus on solution quality. The comparison results are summarized in Table 15. As shown in the table, NuMVC2+p finds better solutions than NuMVC2 on 52 instances while being worse on only 3 instances; FastVC2+p finds better solutions than FastVC2 on 37 instances, while being worse on only 5 instances. This confirms the contribution of the preprocessing algorithm.

\section{Summary and Future Work}

This work explored techniques for solving the MinVC problem in massive sparse graphs, including ideas in construction procedure, local search and preprocessing. We have developed a local search algorithm for MinVC called FastVC, based on two new heuristics. The first heuristic is a construction procedure with a complexity of $O(|E|)$, which strikes a good balance between solution quality and time complexity. The second one is the Best from Multiple Selections (BMS) heuristic, which approximates the minimum loss heuristic very well and lowers the complexity of each removing-vertex selection step from $O(|V|)$ to $O(1)$. Thanks to these two heuristics, the FastVC algorithm performs much better than the state of the art algorithm NuMVC on massive graphs. Experiments on massive real-world graphs show that FastVC finds smaller vertex covers than NuMVC on most graphs.

We also improved previous construction heuristics with ideas in our construction heuristic as well as efficient data structure, and integrated three construction heuristics to improve both NuMVC and FastVC. Furthermore, we have developed a two-phase preprocessing algorithm for MinVC, which is effective and fast. The preprocessing algorithm was applied to improve local search MinVC algorithms, resulting in the NuMVC2+p and FastVC2+p solvers, which further improved the performance on large graphs according to our experiments.

In the future, we would like to design more efficient heuristic algorithms for MinVC as well as other graph problems on massive graphs. We are also interested in applying the ideas in this work to solve large instances of other combinatorial problems. 


\section{Acknowledgement}

This work is supported by National Natural Science Foundation of China 61502464, and 973 Program 2014CB340301. Shaowei Cai is also supported by Youth Innovation Promotion Association, Chinese Academy of Sciences. We would like to thank the anonymous referees for their helpful comments.

\section{References}

Abu-Khzam, F. N., Collins, R. L., Fellows, M. R., Langston, M. A., Suters, W. H., \& Symons, C. T. (2004). Kernelization algorithms for the vertex cover problem: Theory and experiments. In Proceedings of the Sixth Workshop on Algorithm Engineering and Experiments and the First Workshop on Analytic Algorithmics and Combinatorics, pp. 62-69.

Akiba, T., \& Iwata, Y. (2016). Branch-and-reduce exponential/FPT algorithms in practice: A case study of vertex cover. Theor. Comput. Sci., 609, 211-225.

Andrade, D. V., Resende, M. G. C., \& Werneck, R. F. F. (2008). Fast local search for the maximum independent set problem. In Workshop on Experimental Algorithms, pp. 220-234.

Argelich, J., Li, C. M., Manyà, F., \& Planes, J. (2016). The MaxSAT evaluations 2010-2016.. http://www.maxsat.udl.cat.

Barabási, A., \& Albert, R. (1999). Emergence of scaling in random networks. Science, 286(5439), 509.

Cai, S. (2015). Balance between complexity and quality: Local search for minimum vertex cover in massive graphs. In Proceedings of IJCAI 2015, pp. 747-753.

Cai, S., \& Lin, J. (2016). Fast solving maximum weight clique problem in massive graphs. In Proceedings of IJCAI 2016, pp. 568-574.

Cai, S., Lin, J., \& Su, K. (2015). Two weighting local search for minimum vertex cover. In Proceedings of AAAI 2015, pp. 1107-1113.

Cai, S., Su, K., Luo, C., \& Sattar, A. (2013). NuMVC: An efficient local search algorithm for minimum vertex cover. J. Artif. Intell. Res. (JAIR), 46, 687-716.

Cai, S., Su, K., \& Sattar, A. (2011). Local search with edge weighting and configuration checking heuristics for minimum vertex cover. Artif. Intell., 175(9-10), 1672-1696.

Chen, J., Kanj, I. A., \& Jia, W. (2001). Vertex cover: Further observations and further improvements. J. Algorithms, 41(2), 280-301.

Chesler, E. J., \& Langston, M. A. (2005). Combinatorial genetic regulatory network analysis tools for high throughput transcriptomic data. In Systems Biology and Regulatory Genomics, Joint Annual RECOMB 2005 Satellite Workshops on Systems Biology and on Regulatory Genomics, San Diego, CA, USA; December 2-4, 2005, Revised Selected Papers, pp. 150-165.

Dinur, I., \& Safra, S. (2005). On the hardness of approximating minimum vertex cover. Annals of Mathematics, 162(2), 439-486.

Eubank, S., Kumar, V. S. A., Marathe, M. V., Srinivasan, A., \& Wang, N. (2004). Structural and algorithmic aspects of massive social networks. In Proceedings of SODA 2004, pp. 718-727. 
Fang, Z., Chu, Y., Qiao, K., Feng, X., \& Xu, K. (2014). Combining edge weight and vertex weight for minimum vertex cover problem. In Proceedings of FAW 2014, pp. 71-81.

Fomin, F. V., Grandoni, F., \& Kratsch, D. (2009). A measure \& conquer approach for the analysis of exact algorithms. J. ACM, 56(5).

Garey, M., \& Johnson, D. (1979). Computers and Intractability: A Guide to the Theory of NPcompleteness. Freeman, San Francisco, CA, USA.

Hoos, H., \& Stützle, T. (2004). Stochastic Local Search: Foundations and Applications. Morgan Kaufmann, San Francisco, CA, USA.

Jin, Y., \& Hao, J. (2015). General swap-based multiple neighborhood tabu search for the maximum independent set problem. Eng. Appl. of AI, 37, 20-33.

Karakostas, G. (2005). A better approximation ratio for the vertex cover problem. In Proceedings of ICALP 2005, pp. 1043-1050.

Kavalci, V., Ural, A., \& Dagdeviren (2014). Distributed vertex cover algorithms for wireless sensor networks. International Journal of Computer Networks \& Communications (IJCNC), 6, 95110.

Lamm, S., Sanders, P., Schulz, C., Strash, D., \& Werneck, R. F. (2016). Finding near-optimal independent sets at scale. In Proceedings of the Eighteenth Workshop on Algorithm Engineering and Experiments, ALENEX 2016, Arlington, Virginia, USA, January 10, 2016, pp. 138-150.

Li, C. M., \& Huang, W. Q. (2005). Diversification and determinism in local search for satisfiability. In Proceedings of SAT 2005, pp. 158-172.

Lu, L., \& Chung, F. (2006). Complex Graphs and Networks. American Math. Society, New York, USA.

Papadimitrious, C. H., \& Steiglitz, K. (1982). Combinatorial Optimization: Algorithms and Complexity. Prentice Hall, New York, USA.

Pullan, W. (2009). Optimisation of unweighted/weighted maximum independent sets and minimum vertex covers. Discrete Optimization, 6, 214-219.

Richter, S., Helmert, M., \& Gretton, C. (2007). A stochastic local search approach to vertex cover. In Proceedings of KI 2007, pp. 412-426.

Rosin, C. D. (2014). Unweighted stochastic local search can be effective for random CSP benchmarks. CoRR, abs/1411.7480.

Rossi, R. A., \& Ahmed, N. K. (2014). Coloring large complex networks. Social Network Analysis and Mining, 4(1), 228.

Rossi, R. A., \& Ahmed, N. K. (2015). The network data repository with interactive graph analytics and visualization. In Proceedings of AAAI 2015.

Rossi, R. A., Gleich, D. F., Gebremedhin, A. H., \& Patwary, M. M. A. (2014). Fast maximum clique algorithms for large graphs. In WWW (Companion Volume), pp. 365-366.

Selman, B., Levesque, H. J., \& Mitchell, D. G. (1992). A new method for solving hard satisfiability problems. In Proceedings of AAAI 1992, pp. 440-446. 
Strash, D. (2016). On the power of simple reductions for the maximum independent set problem. In Proceedings of COCOON 2016, pp. 345-356.

Verma, A., Buchanan, A., \& Butenko, S. (2015). Solving the maximum clique and vertex coloring problems on very large sparse networks. INFORMS Journal on Computing, 27(1), 164-177.

Wang, Y., Cai, S., \& Yin, M. (2016). Two efficient local search algorithms for maximum weight clique problem. In Proceedings of AAAI 2016, pp. 805-811.

Yadav, T., Sadhukhan, K., \& Rao, A. M. (2016). Approximation algorithm for n-distance minimal vertex cover problem. CoRR, abs/1606.02889. 